Miyazaki, Y.

Osaka J. Math.

28 (1991), 935-973

\title{
THE EIGENVALUE DISTRIBUTION OF ELLIPTIC OPERATORS WITH HÖLDER CONTINUOUS COEFFICIENTS
}

\author{
YO̊ICHI MIYAZAKI
}

(Received March 26, 1991)

\section{Introduction}

In this paper we try to improve the estimates for the remainder term in the asymptotic formula for the eigenvalue distribution of an elliptic operator $A$ of order $2 m$ in $\boldsymbol{R}^{n}$ whose principal part has Hölder continuous coefficients with exponent $\tau, 0<\tau \leqq 1$. In some cases, mainly when $n=1$ we obtain a better estimate for the remainder term of the counting function $N(t)$ than the known results:

$$
N(t)=\mu_{A}(\Omega) t^{n / 2 m}+O\left(t^{(n-\tau) / 2 m}\right) \quad \text { as } t \rightarrow \infty .
$$

In other cases it remains open whether our new estimate is valid or not. Instead of solving this problem we give a corresponding estimate for the remainder term in the asymptotic formula for the partition function (the trace of the heat kernel):

$$
U(t)=\Gamma\left(\frac{n}{2 m}+1\right) \mu_{A}(\Omega) t^{-n / 2 m}+O\left(t^{(\tau-n) / 2 m}\right) \quad \text { as } t \rightarrow 0
$$

when $0<\tau<1$ and $2 m>n$.

In order to describe the results more precisely and compare our results with the known ones we shall recall some standard notations and hypotheses. Let $\Omega$ be a domain in the $n$-dimensional Euclidean space $\boldsymbol{R}^{n}$ with a generic point $x=\left(x_{1}, \cdots, x_{n}\right)$. We denote by $\alpha=\left(\alpha_{1}, \cdots, \alpha_{n}\right) \in \boldsymbol{Z}_{+}^{n}, \boldsymbol{Z}_{+}=\{0,1,2, \cdots\}$ a multi-index of length $|\alpha|=\alpha_{1}+\cdots+\alpha_{n}$ and use the notations

$$
\begin{aligned}
& \partial^{\alpha}=\partial_{x}^{\alpha}=\partial_{1}^{\alpha} \cdots \partial_{n^{n}}^{\alpha}, \quad \partial_{k}=\partial / \partial x_{k}, \\
& D^{\alpha}=D_{x}^{\alpha}=D_{1}^{\alpha} \cdots D_{n^{n}}^{\alpha}, \quad D_{k}=-\sqrt{-1} \partial / \partial x_{k} .
\end{aligned}
$$

For an integer $m \geqq 0 H^{m}(\Omega)$ is to be the set of all functions whose distributional derivatives of order up to $m$ belong to $L_{2}(\Omega)$ and we introduce in it the usual norm 


$$
\|u\|_{m}=\|u\|_{m, \Omega}=\left(\int_{\Omega|\alpha| \leqq m}\left|D^{\alpha} u(x)\right|^{2} d x\right)^{1 / 2}
$$

$H_{0}^{m}(\Omega)$ denotes the closure of $C_{0}^{\infty}(\Omega)$ in $H^{m}(\Omega)$.

For $\tau>0$ we define $\mathscr{B}^{\tau}(\Omega)$ as follows. Let $\tau=k+\sigma$ where $k$ is an integer and $0<\sigma \leqq 1$. $\mathscr{B}^{r}(\Omega)$ is the space of functions $u$ in $\Omega$ such that $\partial^{\alpha} u$ are bounded and continuous for $|\alpha| \leqq k$ and $\left|\partial^{\alpha} u(x)-\partial^{\alpha} u(u)\right| /|x-y|^{\sigma}(x, y \in \Omega$, $x \neq y)$ are bounded for $|\alpha|=k$. We set $\mathscr{B}^{\infty}(\Omega)=\bigcap_{\tau>0} \mathscr{B}^{\tau}(\Omega)$ and denote by $\mathscr{B}^{0}(\Omega)$ the space of bounded continuous functions in $\Omega$.

For the boundary of $\Omega, \Gamma=\partial \Omega$ and $\varepsilon>0$ we define

$$
\Gamma_{\varepsilon}=\{x \in \Omega ; \operatorname{dist}(x, \Gamma)<\varepsilon\}, \quad \widetilde{\Gamma}_{\varepsilon}=\left\{x \in \boldsymbol{R}^{n} ; \operatorname{dist}(x, \Gamma)<\varepsilon\right\} .
$$

Let $V$ be a closed subspace of $H^{m}(\Omega)$ containing $H_{0}^{m}(\Omega)$ :

$$
H_{0}^{m}(\Omega) \subset V \subset H^{m}(\Omega) .
$$

We consider a symmetric integro-differential sesquilinear form $B[u, v]$ of order $m$

$$
B[u, v]=\int_{\alpha} \sum_{|\alpha|,|\beta| \leqq m} a_{\alpha \beta}(x) D^{\alpha} u(x) \overline{D^{\beta} v(x)} d x,
$$

which satisfies the coerciveness:

$$
B[u, v] \geqq \delta\|u\|_{m}^{2}-C_{0}\|u\|_{0}^{2}, \quad \delta>0, C_{0} \geqq 0 \quad \text { for any } u \in V,
$$

and whose coefficients satisfy

$$
a_{\alpha \beta} \in \mathscr{B}^{\top}(\Omega)(|\alpha|=|\beta|=m), \quad a_{\alpha \beta} \in L_{\infty}(\Omega)(|\alpha|+|\beta|<2 m)
$$

for some $\tau>0$.

Let $A$ be the self-adjoint operator associated with this variational triple $\left\{B, V, L_{2}(\Omega)\right\}$, that is, $u \in V$ belongs to $D(A)$, the domain of $A$ if and only if there exists $f \in L_{2}(\Omega)$ such that $B[u, v]=(f, v)$ for any $v \in V$, and we define $A u=f$. Here $($,$) denotes the inner product in L_{2}(\Omega)$.

Let $\Omega$ be bounded. In most cases, for example, when either (1.5) or (1.6) stated below is satisfied, or when $\Omega$ possesses the restricted cone property (see [1]), the spectrum of $A$ consists only of the real eigenvalues $\left\{\lambda_{j}\right\}_{j=1}^{\infty}$ which accumulate only at $\infty$. When we enumerate eigenvalues, we count every eigenvalue as many times as its multiplicity. For a real number $t$ let $N(t)$ be the number of eigenvalues of $A$ not exceeding $t$. Then the well known Weyl's asymptotic formula for $N(t)$ is written

$$
N(t)=\mu_{A}(\Omega) t^{n / 2 m}+O\left(t^{(n-\theta) / 2 m}\right) \quad \text { as } t \rightarrow \infty,
$$

or

(WF2)

$$
N(t)=\mu_{A}(\Omega) t^{n / 2 m}+O\left(t^{(n-1) / 2 m} \log t\right) \quad \text { as } t \rightarrow \infty,
$$


where $\mu_{A}(\Omega)$ is the constant defined in the next section. Formula (WF1) with $\theta=1$ is the best possible if the coefficients and the boundary are sufficiently smooth. We are interested in how large $\theta$ in (WF1) can be taken for a fixed $\tau$ or how small $\tau$ can be taken so that (WF1) holds with $\theta=1$.

When $0<\tau \leqq 1$, Métivier [18] proved (WF1) with $\theta=\tau /(\tau+1)$ under one of the following conditions:

$$
\begin{aligned}
& \text { (1.5) } V=H_{0}^{m}(\Omega) \text { and lim } \sup _{\varepsilon \rightarrow 0}\left|\Gamma_{\varepsilon}\right| / \varepsilon<\infty . \\
& \text { (1.6) } \left.\Omega \text { has }\left(C^{\prime}\right) \text { property (see }[18]\right) \text { and lim } \sup _{\varepsilon \rightarrow 0}\left|\widetilde{\Gamma}_{\varepsilon}\right| / \varepsilon<\infty .
\end{aligned}
$$

Here and in what follows for a Lebesgue measurable set $D$ in $\boldsymbol{R}^{n}$ we denote by $|D|$ its Lebesgue measure.

The author [20] showed that Metivier's result remains valid for any fixed $\tau>0$, assuming that $2 m>n$ and that $\Omega$ possesses the restricted cone property.

When $\tau=\infty$, i.e., when the coefficients are in $C^{\infty}$-class, (WF2) was proved independently by Brüning [3], who assumed $D\left(A^{k}\right) \subset H^{k m}(\Omega)$ for some $k$ with $k m>n$ and $\lim \sup _{\varepsilon \rightarrow 0}\left|\Gamma_{\varepsilon}\right| / \varepsilon<\infty$, and by Tsujimoto [31] who assumed some estimate for the resolvent kernel. Furthermore for the Laplacian $(m=1)$ (WF1) with $\theta=1$ was proved by Seeley [26] $(n=3)$ and Pham The Lai [24] $(n \geqq 1)$ when the boundary $\Gamma$ is sufficiently smooth. For the general operator $(m \geqq 1)$ defined by the boundary conditions Vasil'ev [34] also proved (WF1) with $\theta=1$ (see also Metivier [19]). It is noteworthy that Vasil'ev [34] obtained the second term:

$$
N(t)=\mu_{A}(\Omega) t^{n / 2 m}+b t^{(n-1) / 2 m}+o\left(t^{(n-1) / 2 m}\right) \quad \text { as } t \rightarrow \infty,
$$

where $b$ is a constant, under some assumption on the Hamiltonian flow (see also Ivrii [10] for $m=1$ ).

Looking through the proof of (WF2) by Tsujimoto in detail, we can see that (WF2) is valid for some finite number $\tau$, the value of which is not so smart. Hence there is a gap between (WF1) with $\theta=\tau /(\tau+1)$ for any fixed $\tau>0$ and (WF2) for the finite number $\tau$. Therefore we would like to expect that (WF1) holds with $\theta$ larger than $\tau /(\tau+1)$. In fact in this paper we shall show that (WF1) holds with $\theta=\tau$ for $0<\tau \leqq 1$ in some cases and that (WF2) holds for $\tau=$ 1 in some cases. That is, the following theorem will be derived.

Theorem A. Let $\Omega$ be a bounded domain in $\boldsymbol{R}^{n}$. Let a variational triple $\left\{B, V, L_{2}(\Omega)\right\}$ satisfy (1.1)-(1.4).

(i) When $n=1$ and $0<\tau \leqq 1$, the asymptotic formula (WF1) is valid with $\theta=\tau$.

(ii) We assume that $n \geqq 2$ and that $\Omega$ is a rectangular parallelepiped whose sides are parallel to one of the axes, and that the coefficients of top order $(|\alpha|=|\beta|=m)$ satisfy 


$$
a_{\alpha \beta}(x)= \begin{cases}a_{j}(x) & \left(\alpha=\beta=m e_{j}, \quad j=1, \cdots, n\right) \\ 0 & (\text { otherwise }),\end{cases}
$$

where $e_{j}$ is the unit vector in $\boldsymbol{R}^{n}$ whose jth element is 1 and other elements are 0 , and $a_{j}(x), 1 \leqq j \leqq n$ is a function only in $x_{j}$. Then $(W F 1)$ is valid with $\theta=\tau$ when $0<\tau \leqq 1$.

(iii) We assume that $n \geqq 2, V=H_{0}^{m}(\Omega)$ and $\lim \sup _{\varepsilon \rightarrow 0}\left|\widetilde{\Gamma}_{\varepsilon}\right| / \varepsilon<\infty$, and that the coefficients of top order $(|\alpha|=|\beta|=m)$ satisfy (1.7). Then (WF1) is valid with $\theta=\tau$ when $0<\tau<1$, and (WF2) is valid when $\tau=1$.

Remark. Theorem $\mathrm{A}$ is also valid when $B[u, v]$ has a non-symmetric part of lower terms if $2 m>n$ and $\Omega$ possesses the restricted cone property (see [20, Theorem 2]).

Theorem $A$ is proved in sections 3 and 4. The proof of Theorem A.(i) is based on Theorem 3.2 stated in section 3.1. which gives the remainder estimate of the counting function for the Sturm-Liouville operator $A(m=1)$ :

$$
A u=-\frac{d}{d x}\left(p(x) \frac{d u}{d x}\right), \quad D(A)=\left\{u \in H_{0}^{1}(I) ; A u \in L_{2}(I)\right\}
$$

with $p \in C^{1}(\bar{I})$ where $I$ is a finite open interval in $\boldsymbol{R}$. We will obtain Theorem 3.2 by using a variant of the Prüfer transform which was used by Shimakura [28]. Theorem 3.2 is extended to a $2 m$ th-order operator which has the same differential expression as $A^{m}$ when $p(x)$ is sufficiently smooth (Proposition 3.4). It is important that the remainder term of the asymptotic formula can be estimated by the constant which depends on $\min p(x), \max p(x)$ and $\max \left|p^{\prime}(x)\right|$ and is independent of $\max \left|p^{(k)}(x)\right|, k \geqq 2$. By approximating the Hölder continuous function by smooth functions and using the properties of $N(t, B, V, H)$ stated in section 2.1 we complete the proof of Theorem A.(i) in section 3.3.

Theorem A. (ii) is derived from Theorem A.(i) in section 4.1. Theorem A.(iii) is proved in section 4.2 by using a stronger version of Theorem A.(ii) (Corollary 4.3) and the method of Dirichlet-Neumann bracketing which is originated in Courant-Hilbert [4].

We wonder whether (WF1) with $\theta=\tau \mid(\tau+1)$ for a fixed $\tau, 0<\tau \leqq 1$ or (WF2) for $\tau=1$ are valid or not in the other cases which are not mentioned in Theorem A. This problem remains open. But we will try to give some contribution to this problem. Instead of $N(t)$ we consider the partition function $U(t)$, that is, the trace of the heat kernel $U(t, x, y)$ (the kernel of the semi-group $\left.e^{-t A}\right)$ :

$$
U(t)=\int_{\Omega} U(t, x, x) d x=\int_{-\infty}^{\infty} e^{-t s} d N(s) .
$$

Then we obtain the corresponding result for the partition function, although 
we assume $2 m>n$.

Theorem B. Let $2 m>n$. Let $\Omega$ be a bounded domain with the restricted cone property. Let a variational triple $\left\{B, V, L_{2}(\Omega)\right\}$ satisfy (1.1)-(1.4). Then the following formulas hold.

(i) When $0<\tau<1$, we have

$$
U(t)=\Gamma\left(\frac{n}{2 m}+1\right) \mu_{A}(\Omega) t^{-n / 2 m}+O\left(t^{(\tau-n) / 2 m}\right) \quad \text { as } t \rightarrow 0 .
$$

(ii) When $\tau=1$, we have

$$
U(t)=\Gamma\left(\frac{n}{2 m}+1\right) \mu_{A}(\Omega) t^{-n / 2 m}+O\left(t^{(1-n) / 2 m} \log t^{-1}\right) \quad \text { as } t \rightarrow 0 .
$$

Here $\Gamma(x)$ is the Gamma function.

The proof of Theorem $\mathrm{B}$ is given in section 5, which is independent of sections 3 and 4. In section 5.1 for the operator $A$ with $C^{\infty}$-class coefficients $a_{\alpha \beta}(x)$ in the whole space $\boldsymbol{R}^{n}$ we construct a parametrix for $A-\lambda$ by pseudo-differential operators and estimate the resolvent kernel in terms of the differentiability of the coefficients $a_{\alpha \beta}(x)$. In the remainder estimate of the resolvent kernel the derivatives of higher order of $a_{\alpha \beta}(x)$ appear. Roughly speaking, it is evaluated by the sum of the terms of the form

$$
\text { const } \sup _{x}\left|\partial_{x}^{\gamma} a_{\alpha \beta}(x)\right||\lambda|^{(n-j) / 2 m} .
$$

The point of the proof of Theorem B lies in the fact that $\alpha, \beta, \gamma$ and $j$ vary so that $|\gamma|+(2 m-|\alpha|-|\beta|)-j$ remains constant. The estimate for the heat kernel in $\boldsymbol{R}^{n}$ is derived by the Laplace transform. In section 5.2 we estimate the trace of the heat kernel in a bounded domain $\Omega$ when the coefficients are in $C^{\infty}$-class. In the process we use the estimates for the resolvent kernels by Maruo and Tanabe [14] and Tsujimoto [32]. In section 5.3 approximating the Hölder continuous function by smooth functions and using the properties of $N(t, B, V$, $H$ ) we complete the proof of Theorem B.

As is well known, the asymptotic formula for $N(t)$ is connected with the asymptotic formula for the spectral function $e(t, x, y)$ through the formula

$$
N(t)=\int_{\Omega} e(t, x, x) d x .
$$

It is also interesting to investigate the asymptotic behavior of the spectral function. But we are contented with stating the following. As far as we know, the best results for the spectral function corresponding to (WF1) and (WF2) are

$$
e(t, x, x)=\mu_{A}(x) t^{n / 2 m}+O\left(\delta(x)^{-\theta} t^{(n-\theta) / 2 m}\right) \quad \text { as } t \rightarrow \infty
$$

with $\theta, 0<\theta<\tau /(\tau+2)$ for any fixed $\tau>0$ by Tsujimoto [32], and 


$$
e(t, x, x)=\mu_{A}(x) t^{n / 2 m}+O\left(\delta(x)^{-1} t^{(n-1) / 2 m}\right) \quad \text { as } t \rightarrow \infty
$$

by Brüning [3] and Tsujimoto [31] for $\tau=\infty$, where $\mu_{A}(x)$ is the function defined in the next section and $\delta(x)=\min \{$ dist $(x, \partial \Omega), 1\}$.

In the next section we prepare some lemmas for the proof of Theorems $\mathrm{A}$ and $\mathrm{B}$.

\section{Notation and Preliminaries}

\subsection{Properties of $N(t, B, V, H)$}

Following Métivier [18] we shall state the properties of $N(t, B, V, H)$. Let $H$ and $V$ be two Hilbert spaces with $V \subset H$. Let the inclusion from $V$ into $H$ be continuous . We denote by $(f, g)\left(\operatorname{resp} .(u, v)_{V}\right)$ the inner product in $H$ (resp. $V$ ) and put $\|f\|=(f, f)^{1 / 2}$ and $\|u\|_{V}=(u, u)_{V}^{1 / 2}$. Let $B$ be a continuous symmetric sesquilinear form defined on $V \times V$ :

$$
|B[u, v]| \leqq M\|u\|_{V}\|v\|_{v}, \quad M>0 \quad \text { for any } u, v \in V .
$$

$B$ is said to be strongly coercive on $V$ if there exists $\delta>0$ such that

$$
B[u, u] \geqq \delta\|u\|_{V}^{2} \quad \text { for any } u \in V .
$$

$B$ is said to be coercive on $V$ if there exists $\delta>0$ and $C_{0} \geqq 0$ such that

$$
B[u, u] \geqq \delta\|u\|_{V}^{2}-C_{0}\|u\|^{2} \quad \text { for any } u \in V .
$$

$\{B, V, H\}$ is said to be a variational triple if $V \rightarrow H$ is continuous and $B$ is a continuous symmetric sesquilinear form on $V \times V$ and coercive on $V$. It is convenient to denote a form $B+\mu(),(\mu \in \boldsymbol{R})$ simply by $B+\mu$.

Definition. For a variational triple $\{B, V, H\}$ and $t \in \boldsymbol{R}$ we define $N(t, B, V, H)=\inf \left\{\operatorname{codim}_{V} E ; E\right.$ is a closed subspace of $V$ such that $B-t$ is strongly coercive on $E$ \}.

Lemma 2.1 ([18]).

(i) $N(t+s, B+s, V, H)=N(t, B, V, H)$ for $s \in \boldsymbol{R}$,

(ii) $N(s t, s B, V, H)=N(t, B, V, H)$ for $s>0$.

(iii) If $\left\{B_{1}, V, H\right\},\left\{B_{2}, V, H\right\}$ are two variational triples such that $B_{1}[u, u]$ $\leqq B_{2}[u, u]$ for any $u \in V$, then

$$
N\left(t, B_{1}, V, H\right) \geqq N\left(t, B_{2}, V, H\right) .
$$

(iv) If $\{B, V, H\}$ is a variational triple, and $V_{0}$ is a closed subspace of $V$, then

$$
N\left(t, B, V_{0}, H\right) \leqq N(t, B, V, H) \leqq N\left(t, B, V_{0}, H\right)+N\left(t, B, Z_{t}, H\right),
$$
where $Z_{t}=\left\{u \in V ; B[u, v]=t(u, v)\right.$ for any $\left.v \in V_{0}\right\}$. 
Let $A$ be the self-adjoint operator associated with a variational triple $\{B$, $V, H\}$. Let the inclusion mapping $V \rightarrow H$ be compact. Then the spectrum of $A$ consists of all the eigenvalues of $A$ which accumulate only at $\infty$. The next lemma clarifies the relation between $N(t, B, V, H)$ and $N(t, A)$ which denotes the number of eigenvalues of $A$ not exceeding $t$.

Lemma 2.2 ([18]).

$$
N(t, B, V, H)=N(t, A) .
$$

Lemma 2.3. Let $\Omega$ be a finite open interval I in $\boldsymbol{R}$. Let $\left\{B, V, L_{2}(I)\right\}$ be a variational triple satisfying (1.1)-(1.4). Then we have

$$
\begin{aligned}
N\left(t, B, H_{0}^{m}(I), L_{2}(I)\right) & \leqq N\left(t, B, V, L_{2}(I)\right) \\
& \leqq N\left(t, B, H_{0}^{m}(I), L_{2}(I)\right)+2 m .
\end{aligned}
$$

Proof. Let $Z_{t}=\left\{u \in V ; B[u, v]=t(u, v)\right.$ for any $\left.v \in H_{0}^{m}(I)\right\}$. Because of Lemma 2.1 and because $N\left(t, B, Z_{t}, L_{2}(I)\right) \leqq \operatorname{dim} Z_{t}$, we have only to prove

$$
\operatorname{dim} Z_{t} \leqq 2 m,
$$

which is seen from that $Z_{t}$ is contained in the space of solutions of $2 m$ th-order ordinary differential equation $A u=t u$.

\subsection{Approximation of functions}

Let $0<\tau \leqq 1$. For a sufficiently differentiable function $f$ defined on $\Omega$ we define semi-norms

$$
\begin{aligned}
& |f|_{0}=|f|_{0, \Omega}=\sup \{|f(x)| ; x \in \Omega\}, \\
& |f|_{\tau}=|f|_{\tau, \Omega}=\sup \left\{|f(x)-f(y)| /|x-y|^{\tau} ; x, y \in \Omega, x \neq y\right\} .
\end{aligned}
$$

The following lemma can be easily seen.

Lemma 2.4. Let $\varphi \in C_{0}^{\infty}\left(\boldsymbol{R}^{n}\right)$ satisfy

$$
\int_{\boldsymbol{R}^{n}} \varphi(x) d x=1, \quad \varphi(x) \geqq 0, \quad \operatorname{supp} \varphi \subset\left\{x \in \boldsymbol{R}^{n} ;|x|<1\right\} .
$$

For $\varepsilon>0$ put

$$
\varphi_{\mathrm{g}}(x)=\varepsilon^{-n} \varphi(x / \varepsilon) .
$$

Let $f \in \mathscr{B}^{\tau}\left(\boldsymbol{R}^{n}\right), 0 \leqq \tau \leqq 1$. Then the following inequalities hold.

(i) $\left|\varphi_{\varepsilon} * f\right|_{0} \leqq|f|_{0}$.

(ii) $\left|\varphi_{\varepsilon} * f-f\right|_{0} \leqq|f|_{\tau} \varepsilon^{\tau}$ when $0<\tau \leqq 1$.

(iii) For $\alpha,|\alpha| \geqq 1$ we have

$$
\left|\partial^{\alpha}\left(\varphi_{\varepsilon} * f\right)\right|_{0} \leqq v_{n}\left|\partial^{\alpha} \phi\right|_{0}|f|_{\tau} \varepsilon^{\tau-|\alpha|} .
$$

Here $v_{n}$ denotes the volume of the unit ball in $\boldsymbol{R}^{n}$. 


\subsection{Polynomials with weight}

In this subsection any function is to be sufficiently differentiable if not specified.

In the proof of Theorems $\mathrm{A}$ and $\mathrm{B}$ we encounter polynomials in the derivatives of the coefficients $a_{\alpha \beta}(x)$ in (1.2). We must describe these polynomials precisely so that we can see how they depend on the differentiability of the coeficients $a_{\alpha \beta}(x)$. To this end we introduce polynomials with weight.

Let $n \geqq 1, m \geqq 0$ and $k \geqq 0$ be integers. For multi-indexes $\alpha, \beta \in Z_{+}^{n}$ with $|\alpha| \leqq 2 m$ we consider a polynomial $Q\left(z_{\alpha}^{(\beta)}\right)$ in variables $z_{\alpha}^{(\beta)}$ 's. A polynomial $Q\left(z_{\alpha}^{(\beta)}\right)$ is said to be a polynomial with weight $k$ with respect to $\{n, m ; 1\}$ if $Q\left(z_{\alpha}^{(\beta)}\right)$ can be written

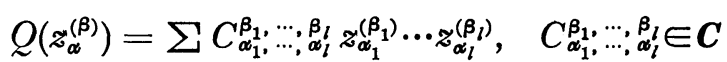

with the finite sum taken over $|\alpha| \leqq 2 m$ and $\left(2 m-\left|\alpha_{1}\right|+\left|\beta_{1}\right|\right)+\cdots+\left(2 m-\left|\alpha_{l}\right|\right.$ $\left.+\left|\beta_{l}\right|\right)=k$. Here $\boldsymbol{C}$ stands for the complex plane. We denote by $\mathscr{F}_{k}\left(=\mathscr{F}_{k, 1}=\right.$ $\left.\mathscr{I}_{k, n, m, 1}\right)$ the set of all polynomials with weight $k$ with respect to $\{n, m ; 1\}$.

Let $A$ be a $2 m$ th-order differential operator such that

$$
A u(x)=\sum_{|\alpha| \leqq 2 m} a_{\alpha}(x) D^{\alpha} u(x) .
$$

Substituting $z_{\alpha}^{(\beta)}=a_{\alpha}^{(\beta)}(x)=\partial_{x}^{\beta} a_{\alpha}(x)$ in (2.1) we obtain the function $Q\left(a_{\alpha}^{(\beta)}(x)\right)$ in $x$, which we denote by $Q[A](x)$.

In particular when $n=1$ and $m=0$ we write $z_{\alpha}^{(\beta)}$ simply by $z^{(\beta)}$ and set

$$
\widetilde{O}[p]=Q\left[A_{p}\right]
$$

for a function $p(x)$ defined on an interval in $\boldsymbol{R}$, where

$$
A_{p} u(x)=p(x) u(x) .
$$

For example the function $p(x) p^{\prime \prime}(x)+3 p^{\prime}(x) p^{\prime}(x)$ is corresponding to the polynomial $z^{(0)} z^{(2)}+3 z^{(1)} z^{(1)}$ with weight 2 .

Next we consider another type of polynomials $Q\left(z_{\alpha \beta}^{(\gamma)}\right)$ in variables $z_{\alpha \beta}^{(\gamma)}$ 's for multi-indexes $\alpha, \beta$ and $\gamma \in Z_{+}^{n}$ with $|\alpha| \leqq m,|\beta| \leqq m$. A polynomial $Q\left(z_{\alpha \beta}^{(\gamma)}\right)$ is said to be a polynomial with weight $k$ with respect to $\{n, m ; 2\}$ if $Q\left(z_{\alpha \beta}^{(\gamma)}\right)$ can be written

$$
Q\left(z_{\alpha \beta}^{(\gamma)}\right)=\sum C_{\alpha_{1} \beta_{1}, \cdots, \alpha_{l} \beta_{l}}^{\gamma_{1}, \cdots, \gamma_{l}} z_{\alpha_{1} \beta_{1}}^{\left(\gamma_{1}\right)} \cdots z_{\alpha_{l} \beta_{l}}^{\left(\gamma_{l}\right)}, \quad C_{\alpha_{1} \beta_{1}, \cdots, \alpha_{l} \beta_{l}}^{\gamma_{1}, \cdots, \gamma_{l}} \in C
$$

with the finite sum taken over $|\alpha| \leqq m,|\beta| \leqq m$ and $\left(2 \mathrm{~m}-\left|\alpha_{1}\right|-\left|\beta_{1}\right|+\left|\gamma_{1}\right|\right)+$ $\cdots+\left(2 m-\left|\alpha_{l}\right|-\left|\beta_{l}\right|+\left|\gamma_{l}\right|\right)=k$. We denote by $\mathscr{F}_{k, 2}\left(=\mathscr{F}_{k, n, m, 2}\right)$ the set of all polynomials with weight $k$ with respect to $\{n, m ; 2\}$.

Let $B$ be an integro-differential sesquilinear form defined by (1.2). Substituting $z_{\alpha \beta}^{(\gamma)}=a_{\alpha \beta}^{(\gamma)}(x)$ in (2.2) we obtain the function $Q\left(a_{\alpha \beta}^{(\gamma)}(x)\right)$ in $x$, which we 
denote by $Q[B](x)$.

The following properties will be often used.

Lemma 2.5. Let $A, B$ and $p$ be as above. Let $\varphi_{\mathrm{g}}$ be the function defined in Lemma 2.4 .

(i) If $Q \in \mathscr{F}_{k, 1}$ (resp. $\left.\mathscr{F}_{k, 2}\right)$ and $R \in \mathscr{F}_{l, 1}$ (resp. $\left.\mathscr{F}_{l, 2}\right)$, then it follows that $Q R$ $\in \mathscr{F}_{k+l, 1}\left(\right.$ resp. $\left.\mathscr{F}_{k+l, 2}\right)$ and that

$$
(Q R)[A]=Q[A] R[A] \quad(\operatorname{resp} .(Q R)[B]=Q[B] R[B]) .
$$

(ii) For a multi-index $\alpha$ and $Q \in \mathscr{F}_{k, 1}\left(\right.$ resp. $\left.\mathscr{F}_{k, 2}\right)$ there exists $R \in \mathscr{F}_{k+|\alpha|, 1}$ (resp. $\mathscr{F}_{k+|\alpha|, 2}$ such that

$$
\partial_{x}^{\alpha} Q[A](x)=R[A](x) \quad\left(\operatorname{resp} . \partial_{x}^{\alpha} Q[B](x)=R[B](x)\right) .
$$

(iii) Suppose that $A$ is associated with $B$, that is, $B[u, v]=(A u, v)$ for $u, v$ $\in C_{0}^{\infty}(\Omega)$. Then for $Q \in \mathscr{F}_{k, 1}$ there exists $R \in \mathscr{F}_{k, 2}$ such that

$$
Q[A](x)=R[B](x) .
$$

(iv) Let $p \in \mathscr{B}^{\tau}(\boldsymbol{R})(0<\tau \leqq 1), Q \in \mathscr{F}_{k, 1}(k \geqq 1)$ and $0<\varepsilon<1$. Then there eixsts $C>0$ independent of $\varepsilon$ such that

$$
\left|Q\left[\varphi_{\varepsilon} * p\right]\right|_{0} \leqq C \varepsilon^{\tau-k} \text {. }
$$

(v) Let $B$ satisfy (1.2) and (1.4) with $\Omega=\boldsymbol{R}^{n}$ and $0<\tau \leqq 1$. Let $Q \in$ $\mathscr{F}_{h, 2}(k \geqq 1)$. For $\varepsilon, 0<\varepsilon<1$ define the form $B_{\varepsilon}$ by replacin $a_{\alpha \beta}(x)$ with $\varphi_{\mathrm{e}} * a_{\alpha \beta}(x)$ in (1.2). Then there exists $C>0$ independent of $\varepsilon$ such that

$$
\left|Q\left[B_{\mathrm{z}}\right]\right|_{0} \leqq C \varepsilon^{r-k} \text {. }
$$

Proof. (i) and (ii) are easily seen. (iii) follows from

$$
a_{\alpha}(x)=\sum_{\beta+\delta=\alpha, \delta \leq \gamma}\left(\begin{array}{l}
\gamma \\
\delta
\end{array}\right) D^{\gamma-\delta} a_{\beta \gamma} .
$$

(iv) and (v) follow from Lemma 2.4, (iii).

\subsection{Miscellaneous}

Lemma 2.6. For $a>0$ and $\lambda \in C \backslash[0, \infty)$ we have

$$
|a-\lambda| \geqq \frac{d(\lambda)}{2|\lambda|}(a+|\lambda|),
$$

where $d(\lambda)=\operatorname{dist}(\lambda, \boldsymbol{C} \backslash[0, \infty))$.

Proof. We put $\lambda=t+\sqrt{-1}$. When $t \leqq 0$, we have

$$
|a-\lambda|=\sqrt{(a+|t|)^{2}+s^{2}} \geqq \frac{1}{\sqrt{2}}(a+|t|+|s|) \geqq \frac{1}{2}(a+|\lambda|),
$$


from which (2.4) follows. When $t>0,(2.4)$ is equivalent to the second-order inequality for $a$ :

$$
\left(4|\lambda|^{2}-s^{2}\right) a^{2}-2|\lambda|\left(4|\lambda| t+s^{2}\right) a+|\lambda|^{2}\left(4|\lambda|^{2}-s^{2}\right) \geqq 0,
$$

which is easily verified.

Q.E.D.

We often use the following interpolation inequality.

Lemma 2.7 ([1]). Let $\Omega$ be a bounded domain in $\boldsymbol{R}^{n}$ with the restricted cone property. Let $0<\varepsilon \leqq 1$ and $k=0, \cdots, m$. Then there exists a constant $C>$ 0 depending only on $m$ and $\Omega$ such that

$$
\|u\|_{k}^{2} \leqq C\left(\varepsilon^{2(m-k)}\|u\|_{2}^{m}+\varepsilon^{-2 k}\|u\|_{0}^{2}\right) \quad \text { for any } u \in H^{m}(\Omega) .
$$

Finally we conclude this section with defining some notations. We often write $N(t, B, V, H)$ simply by $N(t, B, V)$. Let $B$ be the sesquilinear form defined by (1.2) and let $A$ be the differential operator associated with $B$. We set

$$
\begin{aligned}
& a(x, \xi)=\sum_{|\alpha|=|\beta|=m} a_{\alpha \beta}(x) \xi^{\alpha+\beta}, \\
& \mu_{A}(x)=(2 \pi)^{-n} \int_{a(x, \xi)<1} d \xi, \quad \mu_{A}(\Omega)=\mu_{B}(\Omega)=\int_{\Omega} \mu_{A}(x) d x .
\end{aligned}
$$

For a function $f$ defined on $\Omega$ we define

$$
m(f)=m_{\mathbf{\Omega}}(f)=\min \{f(x) ; x \in \Omega\}
$$

\section{Ordinary Differential Operators}

In this section we will prove Theorem A.(i).

\subsection{Sturm-Liouville operators}

Let $I=(a, b)$ be a finite open interval in $\boldsymbol{R}$. For a real-valued function $p \in C^{1}(\bar{I})$ with $0<m(p) \leqq|p|_{0}<\infty$, we consider the self-adjoint operator $A$ in $L_{2}(I)$ defined by

$$
A u=-\frac{d}{d x}\left(p(x) \frac{d}{d x} u\right), \quad D(A)=\left\{u \in H_{0}^{1}(I) ; A u \in L_{2}(I)\right\}
$$

In order to obtain the estimate for $N(t, A)$ we follow Shimakura's method ([28]) which is based on a variant of the Prufer transform. Since this variant of the Prufer transform does not seem to be widely known, we shall review it briefly for sake of convenience.

To solve the eigenvalue problem

$$
A u=\lambda u,
$$


we start with the following initial value problem

$$
\frac{d}{d x}\left(p(x) \frac{d u}{d x}\right)+\lambda u=0, \quad u(a)=0, \quad p(a) u^{\prime}(a)=1 .
$$

Here we may assume $\lambda>0$ because $A$ is a positive operator. The variant of the Prüfer transform given by

$$
\begin{aligned}
p \frac{d u}{d x}+\sqrt{-1} \sqrt{\lambda p} u & =r(x, \lambda) \exp (\sqrt{-1} \theta(x, \lambda)), \\
& \text { with } r(x, \lambda)>0, \quad 0 \leqq \theta(a, \lambda)<2 \pi,
\end{aligned}
$$

which is equivalent to

$$
u=\frac{r}{\sqrt{\lambda p}} \sin \theta, \quad \frac{d u}{d x}=\frac{r}{p} \cos \theta
$$

changes (3.2) into the following equations for $r(x, \lambda)$ and $\theta(x, \lambda)$ :

$$
\begin{array}{ll}
\frac{1}{r} \frac{d r}{d x}=\frac{p^{\prime}}{2 p} \sin ^{2} \theta, & r(a, \lambda)=1, \\
\frac{d \theta}{d x}=\sqrt{\frac{\lambda}{p}}+\frac{p^{\prime}}{4 p} \sin 2 \theta, & \theta(a, \lambda)=0 .
\end{array}
$$

Integrating (3.3) from $a$ to $b$ we obtain

$$
\theta(b, \lambda)-\sqrt{\lambda} \int_{a}^{b} \frac{d x}{\sqrt{p(x)}}=\int_{a}^{b} \frac{p^{\prime}(x)}{4 p(x)} \sin 2 \theta(x, \lambda) d x,
$$

from which it follows that

$$
\left|\theta(b, \lambda)-\sqrt{\lambda} \int_{a}^{b} \frac{d x}{\sqrt{p(x)}}\right| \leqq \frac{\left|p^{\prime}\right|_{0}|I|}{4 m(p)} .
$$

\section{Lemma 3.1.}

(i) $\theta(x, \lambda)$ is a non-decreasing function in $\lambda$ for a fixed $x$.

(ii) If $\theta\left(x_{0}, \lambda\right)=j \pi$ for some $x_{0} \geqq a$ and some integer $j$, then $\theta(x, \lambda)>j \pi$ for $x>x_{0}$ and $\theta(x, \lambda)<j \pi$ for $a \leqq x<x_{0}$.

(iii) The first eigenfunction of $A$ has no zero in the open interval $I=(a, b)$.

Proof. (i) follows from the lemma concerning the differential inequalities ([8, Corollary 4.2, p27]).

(ii) is easily seen from $\theta^{\prime}\left(x_{0}, \lambda\right)=\sqrt{\lambda / p\left(x_{0}\right)}>0$.

(iii) is well known ([9, Theorem 8.3.3, p398]).

Q.E.D.

From Lemma 3.1 it follows that for $\lambda_{k}$, the $k$ th eigenvalue of $A$,

$$
\theta\left(b, \lambda_{k}\right)=k \pi \text {. }
$$


Substituting $\lambda=\lambda_{k}$ in (3.4) and putting $M=\left|p^{\prime}\right|_{0}|I| /(4 \pi m(p))$ we obtain

$$
\left|k-\mu_{A}(I) \sqrt{\lambda_{k}}\right| \leqq M .
$$

First let $t \geqq \lambda_{1}$. Substituting $k=N(t)=N(t, A)$ in (3.5) and noting $\lambda_{N(t)} \leqq t$ we have

$$
N(t) \leqq \mu_{A}(I) t^{1 / 2}+M .
$$

Substituting $k=N(t)+1$ in (3.5) and noting $t<\lambda_{N(t)+1}$ we have

$$
\mu_{A}(I) t^{1 / 2}-M<N(t)+1
$$

Hence it follows that

$$
-M-1<N(t)-\mu_{A}(I) t^{1 / 2} \leqq M \quad \text { for } t \geqq \lambda_{1} .
$$

Next let $0 \leqq t<\lambda_{1}$. From the min-max principle we have

$$
\begin{aligned}
\lambda_{1} & =\min \left\{\int_{I} p(x)\left|u^{\prime}(x)\right|^{2} d x ; u \in H_{0}^{1}(I),\|u\|_{0}=1\right\} \\
& \leqq \min \left\{|p|_{0} \int_{I}\left|u^{\prime}(x)\right|^{2} d x ; u \in H_{0}^{1}(I),\|u\|_{0}=1\right\} \\
& =|p|_{0}\left(\frac{\pi}{|I|}\right)^{2} .
\end{aligned}
$$

Here we have used the fact that the first eigenvalue of $A$ with $p(x) \equiv 1$ is $(\pi /|I|)^{2}$. Since $N(t)=0$, we have for $0 \leqq t<\lambda_{1}$

$$
\left|N(t)-\mu_{A}(I) t^{1 / 2}\right| \leqq \frac{|I|}{\pi \sqrt{m(p)}} \cdot \frac{|p|_{0}^{1 / 2} \pi}{|I|} \leqq\left(\frac{|p|_{0}}{m(p)}\right)^{1 / 2} .
$$

Combining (3.6) and (3.7) we conclude the following.

Theorem 3.2. Let $A$ be the self-adjoint operator defined by (3.1) for $p \in$ $C^{1}(\bar{I})$ with $0<m(p) \leqq|p|_{0}<\infty$. Then we have

$$
\left|N(t, A)-\mu_{A}(I) t^{1 / 2}\right| \leqq \frac{\left|p^{\prime}\right|_{0}|I|}{4 \pi m(p)}+\left(\frac{|p|_{0}}{m(p)}\right)^{1 / 2}+1
$$

for $t \geqq 0$.

REMARK. Instead of Theorem 3.2 Shimakura [28] obtained the estimate for $N(t, A)$ in the following form. The equality

$$
N(t, A)-\left[\mu_{A}(I) t^{1 / 2}\right]=0 \text { or } \pm 1
$$

holds for sufficiently large $t$. 


\subsection{Ordinary differential operators of higher order}

For a real-valued function $p \in C^{\infty}(\bar{I})$ with

$$
0<m(p) \leqq|p|_{0}<\infty,
$$

we define the differential expression $\mathcal{L}_{p}$ by

$$
\mathcal{L}_{p} u=-\frac{d}{d x}\left(p(x) \frac{d u}{d x}\right)
$$

Let $A$ be the self-adjoint operator defined by

$$
A u=\mathcal{L}_{p} u, \quad D(A)=H^{2}(I) \cap H_{0}^{1}(I) .
$$

We note that $A$ is the same operator as defined by (3.1). Then $A^{m}$, the $m$ th power of $A$ satisfies

$$
A^{m} u=\mathcal{L}_{p}^{m} u, \quad H_{0}^{2 m}(I) \subset D\left(A^{m}\right) \subset H^{2 m}(I) .
$$

On the other hand, we define the self-adjoint operator $A_{m}$ by

$$
A_{m} u=\mathcal{L}_{p}^{m} u, \quad D\left(A_{m}\right)=H^{2 m}(I) \cap H_{0}^{m}(I),
$$

and also have

$$
H_{0}^{2 m}(I) \subset D\left(A_{m}\right) \subset H^{2 m}(I) .
$$

In order to compare $N\left(t, A^{m}\right)$ and $N\left(t, A_{m}\right)$ we consider the sesquilinear form

$$
b[u, v]=\int_{I} \mathcal{L}_{p}^{m} u \overline{\mathcal{L}_{p}^{m} v} d x .
$$

It is easily seen from (3.8) and the interpolation inequality that $b$ is coercive. We note that $\left(A^{m}\right)^{2}$ (resp. $\left.\left(A_{m}\right)^{2}\right)$ is an operator associated with the variational triple $\left\{b, D\left(A^{m}\right), L_{2}(I)\right\}$ (resp. $\left.\left\{b, D\left(A_{m}\right), L_{2}(I)\right\}\right)$. From Lemma 2.3 we have

$$
\left|N\left(t, b, D\left(A^{m}\right)\right)-N\left(t, b, D\left(A_{m}\right)\right)\right| \leqq 4 m,
$$

that is,

$$
\left|N\left(t,\left(A^{m}\right)^{2}\right)-N\left(t,\left(A_{m}\right)^{2}\right)\right| \leqq 4 m
$$

which gives

$$
\left|N\left(t, A^{m}\right)-N\left(t, A_{m}\right)\right| \leqq 4 m .
$$

In what follows until the end of this section we write $\mathscr{F}_{k, 1,0,1}$ (see section 2.3) simply by $\mathscr{I}_{k}$.

Lemma 3.3. For an integer $m \geqq 1$ there exist polynomials $Q_{i j}\left(z^{(\beta)}\right), 0 \leqq i, j$ 
$\leqq m$ with respect to $\{1,0 ; 1\}$ satisfying the following properties (i) and (ii).

(i) $Q_{i j} \in \mathscr{F}_{2 m-(i+j)}, \bar{Q}_{j i}=Q_{i j}, Q_{m m}\left(z^{(\beta)}\right)=\left(z^{(0)}\right)^{m}$.

(ii) For a real-valued function $p \in C^{\infty}(\bar{I})$ we define the sesquilinear form $B_{m, p}$ by

$$
B_{m, p}[u, v]=\int_{I} \sum_{i, j=0}^{m} Q_{i j}[p](x) D^{i} u(x) \overline{D^{j} v(x)} d x .
$$

Then it follows that

$$
B_{m, p}[u, v]=\left(\mathcal{L}_{p}^{m} u, v\right) \quad \text { for any } u \in H^{2 m}(I), v \in H_{0}^{m}(I) .
$$

REMARK. From Lemma 3.3 it follows that $B_{m, p}[u, v]$ is symmetric and that $Q_{m m}[p](x)=p(x)^{m}$.

Proof. For sake of simplicity we write $\mathcal{L}_{p}$ simply by $\mathcal{L}$.

Step 1. First we prove that $\mathcal{L}^{m}$ can be written

$$
\mathcal{L}^{m}=\sum_{j=0}^{2 m} a_{m, j}\left(\frac{d}{d x}\right)^{j}
$$

where $a_{m, j}$ is a real-valued function satisfying

$$
a_{m, j}=Q_{m, j}[p], \quad Q_{m, j} \in \mathscr{F}_{2 m-j}, \quad a_{m, 2 m}=(-1)^{m} p^{m} .
$$

We proceed by induction on $m$. When $m=1,(3.11)$ is seen from

$$
\mathcal{L}=-p\left(\frac{d}{d x}\right)^{2}-p^{\prime} \frac{d}{d x}
$$

Suppose that (3.11) is valid for $m$. Then for $m+1$ we have

$$
\begin{aligned}
\mathcal{L}^{m+1}=\left(-p\left(\frac{d}{d x}\right)^{2}-p^{\prime} \frac{d}{d x}\right) & \left(\sum_{j=0}^{2 m} a_{m, j}\left(\frac{d}{d x}\right)^{j}\right) \\
=-\sum_{j=0}^{2 m}\left\{p a_{m, j}\left(\frac{d}{d x}\right)^{j+2}+\right. & \left(2 p a_{m, j}^{\prime}+p^{\prime} a_{m, j}\right)\left(\frac{d}{d x}\right)^{j+1} \\
& \left.+\left(p a_{m, j}^{\prime \prime}+p^{\prime} a_{m, j}^{\prime}\right)\left(\frac{d}{d x}\right)^{j}\right\},
\end{aligned}
$$

from which it is seen that (3.11) is valid for $m+1$. Hence (3.11) has been proved for any $m \geqq 1$.

Step 2. Let $T$ be a formally self-adjoint differential expression of order $2 m$ such that

$$
T u=\sum_{j=0}^{2 m} a_{j}\left(\frac{d}{d x}\right)^{j} u
$$

where $a_{j}$ is a real-valued function satisfying 


$$
a_{j}=Q_{j}[p], \quad Q_{j} \in \mathscr{F}_{2 m-j+k}
$$

for some nonnegative integer $k$. Then $T$ can be written

$$
T u=\sum_{j=0}^{m}\left(b_{j} u^{(j)}\right)^{(j)},
$$

where $b_{j}$ is a real-valued function satisfying

$$
b_{j}=Q_{j}[p], \quad Q_{j} \in \mathscr{F}_{2 m-2 j+k}, \quad b_{m}=a_{2 m} .
$$

We will prove this assertion by induction on $m$. It is clear when $m=0$.

When $m \geqq 1$, we put

$$
S u=T u-\left(a_{2 m} u^{(m)}\right)^{(m)} .
$$

Then $S$ is formally self-adjoint and can be written

$$
S u=\sum_{j=0}^{2 m-1} c_{j} u^{(j)},
$$

where $c_{j}$ is a real-valued function satisfying

$$
c_{j}=R_{j}[p], \quad R_{j} \in \mathscr{F}_{2 m-j+k} .
$$

Because $S$ is formally self-adjoint and because $c_{2 m-1}$ is real-valued, it follows that $c_{2 m-1}=0$. Hence $S$ is a formally self-adjoint differential expression of order $2 m-2$ with real-valued coefficients satisfying the property corresponding to (3.12) with $m$ replaced by $m-1$ and $k$ replaced by $k+2$. Thus the proof of (3.12) is reduced to the case of $m-1$. Consequently the assertion has been proved.

Step 3. Combining the assertions obtained in step 1 and step 2 and noting $D=-\sqrt{-1} d / d x$, we get Lemma 3.3. $\quad$ Q.E.D.

Proposition 3.4. For an integer $m \geqq 1$ and a real-valued function $p \in C^{\infty}(\bar{I})$ with (3.8) let $B_{m, p}$ be the symmetric sesquilinear form defined in Lemma 3.3. Let $V$ be a closed subspace of $H^{m}(I)$ containing $H_{0}^{m}(I)$. Then $B_{m, p}$ is coercive on $V$ and we have

$$
\left|N\left(t, B_{m, p}, V\right)-\mu_{B_{m, p}}(I) t^{1 / 2 m}\right| \leqq \frac{\left|p^{\prime}\right|_{0}|I|}{4 \pi m(p)}+\left(\frac{|p|_{0}}{m(p)}\right)^{1 / 2}+6 m+1
$$

for $t \geqq 0$.

Proof. The coerciveness of $B_{m, p}$ follows from (3.8) and the interpolation inequality. From Lemma 3.3 it follows that $A_{m}$ defined by (3.9) is the operator associated with $\left\{B_{m}, H_{0}^{m}(I), L_{2}(I)\right\}$. Because of Lemma 2.3 we have

$$
\left|N\left(t, B_{m}, V\right)-N\left(t, A_{m}\right)\right| \leqq 2 m .
$$


This combined with (3.10) gives

$$
\left|N\left(t, B_{m}, V\right)-N\left(t, A^{m}\right)\right| \leqq 6 m .
$$

Noting $N\left(t, A^{m}\right)=N\left(t^{1 / m}, A\right)$ and $\mu_{B_{m, p}}(I)=\mu_{A}(I)$ and using Theorem 3.2 and (3.13) we get the desired result.

Q.E.D.

\subsection{Proof of Theorem A.(i).}

Now we shall prove Theorem A.(i). Let $0<\tau \leqq 1$. Let $\Omega$ be a finite open interval $I$ in $\boldsymbol{R}$. Let $V$ be a closed subspace of $H^{m}(I)$ containing $H_{0}^{m}(I)$. We consider a symmetric sesquilinear form

$$
B[u, v]=\int_{I} \sum_{i, j \unlhd^{m}} a_{i j}(x) D^{i} u(x) \overline{D^{j} v(x)} d x,
$$

which satisfies the coerciveness:

$$
B[u, u] \geqq \delta\|u\|_{m}^{2}-C_{0}\|u\|_{0}^{2}, \quad \delta>0, C_{0} \geqq 0 \quad \text { for any } u \in V,
$$

and whose coefficients satisfy

$$
a_{m m}=p \in \mathscr{B}^{r}(I), \quad a_{i j} \in L_{\infty}(I)(i+j<2 m) .
$$

It is seen from (3.14) and the inverse of Garding's inequality that

$$
0<m(p) \leqq|p|_{0}<\infty .
$$

From the lemma on the extension of the Hölder continuous function ([29, p174]) we may assume that $p \in \mathscr{B}^{T}(\boldsymbol{R})$ and that (3.16) holds for this extended function p. Using the function $\varphi_{\varepsilon}$ defined in Lemma 2.4, we define

and have

$$
q=p^{1 / m}, \quad q_{\mathrm{s}}=\varphi_{\mathrm{e}} * q,
$$

$$
q \in \mathscr{B}^{\top}(\boldsymbol{R}), \quad q_{\mathrm{\varepsilon}} \in C^{\infty}(\boldsymbol{R}) .
$$

From Lemma 2.4 it follows that there exists $\varepsilon_{0}, 0<\varepsilon_{0}<1$ such that

$$
m\left(q_{\varepsilon}\right) \geqq \frac{1}{2} m(q), \quad\left|q_{\varepsilon}\right|_{0} \leqq|q|_{0}
$$

hold for any $\varepsilon, 0<\varepsilon<\varepsilon_{0}$. We define $B_{\varepsilon}$ by

$$
B_{\varepsilon}[u, v]=\int_{I} \sum_{i, j=1}^{m} Q_{i j}\left[q_{\varepsilon}\right](x) D^{i} u(x) \overline{D^{j} v(x)} d x,
$$

where $Q_{i j}$ is the polynomial determined in Lemma 3.3.

In what follows until the end of this section we denote by $C$ constants independent of $\varepsilon, t$ and $u$ which may differ from each other. Let $0<\varepsilon<\varepsilon_{0}$. 
Using Lemma 2.4, Lemma 2.5, Lemma 2.7 and (3.14) we have

$$
\begin{aligned}
\left|B[u, u]-B_{\varepsilon}[u, u]\right| \\
\quad \leqq\left|q^{m}-q_{\varepsilon}^{m}\right|_{0}\|u\|_{m}^{2}+\sum_{i+j<2 m}\left(\left|a_{i j}\right|_{0}+\left|Q_{i j}\left[q_{\mathrm{g}}\right]\right|_{0}\right)\left\|D^{i} u\right\|_{0}\left\|D^{j} u\right\|_{0} \\
\quad \leqq C \varepsilon^{\tau}\|u\|_{m}^{2}+C \sum_{i+j<2 m} \varepsilon^{\tau+i+j-2 m}\|u\|_{i}\|u\|_{j} \\
\quad \leqq C \varepsilon^{\tau}\|u\|_{m}^{2}+C \sum_{i+j<2 m} \frac{1}{2}\left(\varepsilon^{\tau+2 i-2 m}\|u\|_{i}^{2}+\varepsilon^{\tau+2 j-2 m}\|u\|_{j}^{2}\right) \\
\quad \leqq C\left(\varepsilon^{\tau}\|u\|_{m}^{2}+\varepsilon^{\tau-2 m}\|u\|_{0}^{2}\right) \\
\quad \leqq C \varepsilon^{\tau} B[u, u]+C \varepsilon^{\tau-2 m}\|u\|_{0}^{2} .
\end{aligned}
$$

Noting that $1-C \varepsilon^{\tau} \geqq 1 / 2$ if $\varepsilon_{0}$ is sufficiently small, we get

$$
\begin{aligned}
& B[u, u] \geqq\left(1+C \varepsilon^{\tau}\right)^{-1}\left\{B_{\varepsilon}[u, u]-C \varepsilon^{\tau-2 m}\|u\|_{0}^{2}\right\}, \\
& B[u, u] \leqq\left(1-C \varepsilon^{\tau}\right)^{-1}\left\{B_{\varepsilon}[u, u]+C \varepsilon^{\tau-2 m} \|\left. u\right|_{0} ^{2}\right\} .
\end{aligned}
$$

We choose $t_{0}>0$ so that $t_{0}^{-1 / 2 m}=\varepsilon_{0}$. Putting $\varepsilon=t^{-1 / 2 m}$ for $t>t_{0}$ and using (3.19), Lemma 2.1, Proposition 3.4 and Lemma 2.4 we obtain

$$
\begin{aligned}
N(t, & B, V) \\
& \leqq N\left(\left(1+C \varepsilon^{\tau}\right) t+C \varepsilon^{\tau-2 m}, B_{\varepsilon}, V\right) \\
& \leqq \mu_{B_{\varepsilon}}(I)\left\{\left(1+C \varepsilon^{\tau}\right) t+C \varepsilon^{\tau-2 m}\right\}^{1 / 2 m}+\frac{\left|q_{\varepsilon}^{\prime}\right|_{0}|I|}{4 \pi m\left(q_{\varepsilon}\right)}+\left(\frac{\left|q_{\varepsilon}\right|_{0}}{m\left(q_{\varepsilon}\right)}\right)^{1 / 2}+6 m+1 \\
& \leqq \mu_{B}(I)\left(1+C \varepsilon^{\tau}\right)\left\{\left(1+C \varepsilon^{\tau}\right)+C \varepsilon^{\tau-2 m} t^{-1}\right\}^{1 / 2 m} t^{1 / 2 m}+C \varepsilon^{\tau-1} \\
& \leqq \mu_{B}(I) t^{1 / 2 m}+C t^{(1-\tau) / 2 m}
\end{aligned}
$$

for $t>t_{0}$. Similarly we get the estimate for $N(t, B, V)$ from below. Hence we obtain

$$
\left|N(t, B, V)-\mu_{B}(I) t^{1 / 2 m}\right| \leqq C t^{(1-\tau) / 2 m} \quad \text { for } t>t_{0} .
$$

Thus we complete the proof of Theorem A.(i).

For the proof of Theorem A.(iii) we need a stronger version of Theorem A.(i).

Corollary 3.5. Let $\tau, \Omega, V$ and $B$ be defined as in the beginning of this subsection. Put

$$
M=\left|a_{m m}\right|_{\tau}+\sum_{i, j \leqq m}\left|a_{i j}\right|_{0}+m\left(a_{m m}\right)^{-1} .
$$

Then there exists a constant $C>0$ which depends only on $n, m, \Omega$ and $M$, and is an increasing function in $M$ such that

$$
\left|N(t, B, V)-\mu_{B}(I) t^{1 / 2 m}\right| \leqq C\left(1+t^{(1-\tau) / 2 m}\right)
$$


holds for $t \geqq 0$.

Proof. Let us take $t_{0}>0$ as above. Noting that $\delta$ and $C_{0}$ in (3.14) are determined by $M$ and looking through the above proof of Theorem A.(i), we can see that (3.20) holds for $t>t_{0}$ with the constant $C>0$ having the properties stated in the corollary.

When $0 \leqq t \leqq t_{0}$, it follows from Lemmas 2.1, 2.3 and (3.14) that

$$
\begin{aligned}
N(t, B, V) & \leqq N\left(t, B, H_{0}^{m}(I)\right)+2 m \\
& \leqq N\left(\delta^{-1}\left(t_{0}+C_{0}\right), B_{0}, H_{0}^{m}(I)\right)+2 m,
\end{aligned}
$$

where $B_{0}$ is the form defined by

$$
B_{0}[u, v]=\int_{I} \sum_{i=0}^{m} D^{i} u(x) \overline{D^{i} v(x)} d x .
$$

Hence the left-hand side of (3.20) is evaluated by the constant having the properties stated in the corollary when $0 \leqq t \leqq t_{0}$. Thus we obtain the corollary.

\section{Multi-dimensional Operators}

In this section we consider the case of $n \geqq 2$.

\subsection{Proof of Theorem A.(ii).}

Let $0<\tau \leqq 1$. Let $\Omega=I_{1} \times \cdots \times I_{n}$ where $I_{j}, 1 \leqq j \leqq n$ is a finite open interval in $\boldsymbol{R}$. Let $V$ be $H^{m}(\Omega)$ or $H_{0}^{m}(\Omega)$. We consider a sesquilinear form

$$
B[u, v]=\int_{\Omega} \sum_{j=1}^{n} p_{j}\left(x_{j}\right) D_{j}^{m} u(x) \overline{D_{j}^{m} v(x)} d x,
$$

where $p_{j}$ is a function on $\boldsymbol{R}$ and satisfies

$$
p_{j} \in \mathscr{B}^{\top}(\boldsymbol{R}), \quad 0<m\left(p_{j}\right) \leqq\left|p_{j}\right|_{0}<\infty .
$$

Clearly $B$ is coercive on $H^{m}(\Omega)$. Let $A$ be the operator associated with $\{B, V$, $\left.L_{2}(\Omega)\right\}$.

In order to find the eigenvalues of $A$ we consider the form in one dimension

$$
B_{j}[u, v]=\int_{I_{j}} p_{j}(t) D_{t}^{m} u(t) \overline{D_{t}^{m} v(t)} d t \quad \text { on } V_{j},
$$

where $V_{j}$ is $H^{m}\left(I_{j}\right)$ or $H_{0}^{m}\left(I_{j}\right) . \quad B_{j}$ is also coercive on $V_{j}$. Let $A_{j}$ be the operator associated with the variational triple $\left\{B_{j}, V_{j}, L_{2}\left(I_{j}\right)\right\}$. Let $\left\{\lambda_{j, k}\right\}_{k=1}^{\infty}$ and $\left\{\phi_{j, k}\right\}_{k=1}^{\infty}$ be the eigenvalues and the corresponding eigenfunctions of $A_{j}$. Since $A_{j}$ is nonnegative, $N\left(t, A_{j}\right)$ is a non-decreasing function with $N\left(t, A_{j}\right)=0$ for $t<0$.

For non-decreasing functions $N_{i}(t), i=1,2$ defined on $\boldsymbol{R}$ with $N_{i}(t)=0$ for 
$t<0$, we define

$$
N_{1} \# N_{2}(t)= \begin{cases}\int_{[0, t]} N_{1}(t-s) d N_{2}(s) & (t \geqq 0) \\ 0 & (t<0) .\end{cases}
$$

By definition it is clear that $N_{1} \# N_{2}(t)$ is also a non-decreasing function with $N_{1} \# N_{2}(t)=0$ for $t<0$.

Lemma 4.1. In addition to the above situation we assume that $V=H^{m}(\Omega)$ and $V_{j}=H^{m}\left(I_{j}\right), 1 \leqq j \leqq n$. Then the set of the eigenvalues of $A$ consists only of $\left\{\lambda_{1, k_{1}}+\cdots+\lambda_{n, k_{n}} ; k_{j}=1,2, \cdots(1 \leqq j \leqq n)\right\}$.

Moreover it follows that

$$
N(\cdot, A)=N\left(\cdot, A_{1}\right) \# N\left(\cdot, A_{2}\right) \# \cdots \#\left(\cdot, A_{n}\right) .
$$

The same statement is also valid when we replace $H^{m}$ by $H_{0}^{m}$.

Proof. We shall prove Lemma 4.1 only in the case $V=H^{m}(\Omega)$ and $V_{j}=$ $H^{m}\left(I_{j}\right), 1 \leqq j \leqq n$. The proof in the case $V=H_{0}^{m}(\Omega)$ and $V_{j}=H_{0}^{m}\left(I_{j}\right), 1 \leqq j \leqq n$ is carried out similarly. Let

$$
\begin{aligned}
& \hat{I}_{j}=I_{1} \times \cdots I_{j-1} \times I_{j+1} \times I_{n}, \\
& \hat{x}_{j}=\left(x_{1}, \cdots, x_{j-1}, x_{j+1}, \cdots, x_{n}\right) .
\end{aligned}
$$

For a function $u(x)$ such that

$$
u(x)=u_{1}\left(x_{1}\right) \cdots u_{n}\left(x_{n}\right),
$$

where $u_{j}, 1 \leqq j \leqq n$ is a function on $\boldsymbol{R}$, we define the function $\hat{u}_{j}$ in $\hat{x}_{j}$ by

$$
u(x)=u_{j}\left(x_{j}\right) u_{j}\left(\hat{x}_{j}\right)
$$

For sake of simplicity we write

$$
v\left(\cdot, \hat{x}_{j}\right)=v\left(x_{1}, \cdots, x_{j-1}, \cdot, x_{j+1}, \cdots, x_{n}\right) .
$$

For a multi-index $k=\left(k_{1}, \cdots, k_{n}\right)$ we put

$$
\varphi_{k}(x)=\varphi_{1, k_{1}}\left(x_{1}\right) \cdots \varphi_{n, k_{n}}\left(x_{n}\right),
$$

which is clearly an element of $H^{m}(\Omega)$. Because $\varphi_{j, k_{j}}$ is an eigenfunction of $A_{j}$, it follows that for $v \in C^{\infty}\left(\boldsymbol{R}^{n}\right)$,

$$
\begin{aligned}
B\left[\phi_{k}, v\right] & =\sum_{j=1}^{n} \int_{\hat{I}_{j}}\left(\hat{\varphi}_{k}\right)_{j}\left(\hat{x}_{j}\right) B_{j}\left[\varphi_{j, k_{j}}, v\left(\cdot, \hat{x}_{j}\right)\right] d \hat{x}_{j} \\
& =\sum_{j=1}^{n} \lambda_{j, k_{j}} \int_{\hat{I}_{j}}\left(\hat{\varphi}_{k}\right)_{j}\left(\hat{x}_{j}\right) d \hat{x}_{j} \int_{I_{j}} \varphi_{j, k_{j}}\left(x_{j}\right) \overline{v(x)} d x_{j} \\
& =\left(\lambda_{1, k_{1}}+\cdots+\lambda_{n, k_{n}}\right)\left(\varphi_{k}, v\right) .
\end{aligned}
$$


Since $C^{\infty}\left(\boldsymbol{R}^{n}\right)$ is dense in $H^{m}(\Omega),(4.4)$ holds also for any $v \in H^{m}(\Omega)$. Therefore $\varphi_{k} \in D(A)$, and

$$
A \varphi_{k}=\left(\lambda_{1, k_{1}}+\cdots+\lambda_{n, k_{n}}\right) \varphi_{k}
$$

Because of the completeness of the eigenfunctions $\left\{\phi_{j, k}\right\}_{k=1}^{\infty}$ in $L_{2}\left(I_{j}\right)$ the set of the eigenvalues of $A$ consists only of $\left\{\lambda_{1, k_{1}}+\cdots+\lambda_{n, k_{n}}\right\}$. Thus the first half of Lemma 4.1 has been proved.

Next we shall prove (4.3). For a finite set $J$ we denote the cardinality of $J$ by $|J|$. Since $\lambda_{j, k} \geqq 0$, we have only to prove (4.3) for $t \geqq 0$.

$$
\begin{aligned}
\left|\left\{(n, m) ; \lambda_{1, n}+\lambda_{2, m} \leqq t\right\}\right| & =\sum_{s}\left|\left\{m ; \lambda_{2, m}=s\right\}\right| \times\left|\left\{n ; \lambda_{1, n} \leqq t-s\right\}\right| \\
& =\sum_{s}\left(N\left(s, A_{2}\right)-N\left(s-0, A_{2}\right)\right) N\left(t-s, A_{1}\right) \\
& =\int_{[0, t]} N\left(t-s ; A_{1}\right) d N\left(s ; A_{2}\right) .
\end{aligned}
$$

Repeated use of the same calculation as above leads us to (4.3).

Q.E.D.

We prepare the following lemma to evaluate $N(t, A)$ from the estimates for $N\left(t, A_{j}\right), 1 \leqq j \leqq n$ obtained by Theorem A.(i).

Lemma 4.2. Let $N_{i}(t), i=1,2$ be a non-decreasing function defined on $\boldsymbol{R}$ with $N_{i}(t)=0$ for $t<0$. Suppose that there exist positive constants $c_{i}, b_{i}, \theta_{i}, \sigma$ with $\theta_{i} \geqq \sigma>0$ and a function $\gamma_{i}(t)$ such that

$$
N_{i}(t)=c_{i} t^{\theta_{i}}+\gamma_{i}(t), \quad\left|\gamma_{i}(t)\right| \leqq b_{i}\left(t^{\theta_{i}-\sigma}+1\right)
$$

hold for $t \geqq 0$. Then we have

$$
\begin{aligned}
& \left|N_{1} \# N_{2}(t)-c_{1} c_{2} \frac{\Gamma\left(\theta_{1}+1\right) \Gamma\left(\theta_{2}+1\right)}{\Gamma\left(\theta_{1}+\theta_{2}+1\right)} t^{\theta_{1}+\theta_{2}}\right| \\
& \leqq 4\left(b_{1} c_{2}+b_{2} c_{1}+b_{1} b_{2}\right)\left(t^{\theta_{1}+\theta_{2}-\sigma}+1\right) \quad \text { for } t \geqq 0 .
\end{aligned}
$$

Proof. Let $t \geqq 0$. By integration by parts we have

$$
\begin{aligned}
\int_{[0, t]} & N_{2}(t-s) d N_{1}(s) \\
= & c_{1} c_{2} \int_{0}^{t}(t-s)^{\theta_{1}} d\left(s^{\theta_{2}}\right) \\
& \quad+c_{2} \int_{[0, t]} \gamma_{1}(t-s) d\left(s^{\theta_{2}}\right)+\int_{[0, t]} \gamma_{2}(t-s) d N_{1}(s) \\
= & c_{1} c_{2} \frac{\Gamma\left(\theta_{1}+1\right) \Gamma\left(\theta_{2}+1\right)}{\Gamma\left(\theta_{1}+\theta_{2}+1\right)} t^{\theta_{1}+\theta_{2}}+J_{1}+J_{2} .
\end{aligned}
$$

Since generally for $\rho>0$ it follows that $t^{\rho} \leqq 1$ when $0 \leqq t \leqq 1$, and $1 \leqq t^{\rho}$ when 
$1 \leqq t$, we have

$$
\left|J_{1}\right| \leqq b_{1} c_{2}\left(t^{\theta_{1}-\sigma}+1\right) t^{\rho_{2}} \leqq 2 b_{1} c_{2}\left(t^{\theta_{1}+\theta_{2}-\sigma}+1\right)
$$

and

$$
\begin{aligned}
\left|J_{2}\right| & \leqq b_{2}\left(t^{\theta_{2}-\sigma}+1\right) N_{1}(t) \\
& \leqq b_{2}\left(t^{\theta_{2}-\sigma}+1\right)\left(c_{1} t^{\theta_{1}}+b_{1} t^{\theta_{1}-\sigma}+b_{1}\right) \\
& \leqq b_{2}\left(2 c_{1}+4 b_{1}\right)\left(t^{\theta_{1}+\theta_{2}-\sigma}+1\right) .
\end{aligned}
$$

Combining the above equality and inequalities we obtain the desired result.

Q.E.D.

From Theorem A.(i) we have

$$
\left|N\left(t, A_{j}\right)-\mu_{A_{j}}(\Omega) t^{1 / 2 m}\right| \leqq C\left(1+t^{(1-\tau) / 2 m}\right), \quad 1 \leqq j \leqq n
$$

for $t \geqq 0$. Using Lemma 4.2 repeatedly with (4.5) we obtain

$$
\left|N(t, B, V)-\mu_{B}(\Omega) t^{n / 2 m}\right| \leqq C\left(1+t^{(n-\tau) / 2 m}\right) \quad \text { for } t \geqq 0,
$$

where $V=H^{m}(\Omega)$ or $H_{0}^{m}(\Omega)$. From Lemma $2.1(4.6)$ is also valid for any $V$ satisfying (1.1).

Now we are ready to prove Theorem A.(ii). From [17, Proposition 2.1] or $[20$, Lemma 4.3$]$ we may assume that the coefficients of lower order $(|\alpha|+$ $|\beta|<2 m)$ are identically zero. It is seen from the inverse of Garding's inequality and (1.3) that (4.2) is satisfied. Therefore we have only to prove (WF1) for a symmetric form $B$ satisfying (4.1) and (4.2), which is (4.6) itself. Thus we complete the proof of Theorem A.(ii).

Looking through the above proof and using Corollary 3.5 instead of Theorem A.(i), we obtain a stronger version of Theorem A.(ii).

Corollary 4.3. Let $\tau, \Omega$ and $B$ be defined as in the beginning of this subsection and let $V$ satisfy (1.1). Put

$$
M=\sum_{j=1}^{n}\left(\left|p_{j}\right|_{\tau}+\left|p_{j}\right|_{0}+m\left(p_{j}\right)^{-1}\right) .
$$

Then there exists a constant $C>0$ which depends only on $n, m, \Omega$ and $M$, and is an increasing function in $M$ such that

$$
\left|N(t, B, V)-\mu_{B}(\Omega) t^{n / 2 m}\right| \leqq C\left(1+t^{(n-\tau) / 2 m}\right)
$$

holds for $t \geqq 0$.

\subsection{Proof of Theorem A.(iii)}

Let $0<\tau \leqq 1$. Let $\Omega$ be a bounded domain satisfying $\lim \sup _{\mathrm{z} \rightarrow 0}\left|\widetilde{\Gamma}_{\mathrm{z}}\right| / \varepsilon<$ $\infty$. Let $V=H_{0}^{m}(\Omega)$. We consider a symmetric form 


$$
B[u, v]=\int_{R^{n}} \sum_{j=1}^{n} p_{j}\left(x_{j}\right) D_{j}^{m} u(x) \overline{D_{j}^{m} v(x)} d x
$$

with

$$
p_{j} \in \mathscr{B}^{\top}(\boldsymbol{R}), \quad 0<m\left(p_{j}\right) \leqq\left|p_{j}\right|_{0}<\infty .
$$

We note that $B$ can be regarded as a form defined on $H^{m}\left(\Omega^{\prime}\right)$ for any open set $\Omega^{\prime}$ in $\boldsymbol{R}^{n}$.

Lemma 4.4. For $x_{0} \in \boldsymbol{R}^{n}$ and $\varepsilon, 0<\varepsilon \leqq 1$ let $Q_{\varepsilon}\left(x_{0}\right)$ be the cube of side $\varepsilon$ with center $x_{0}$ whose sides are parallel to one of the axes. Then there exists $C>0$ independent of $\varepsilon, x_{0}$ and $t$ such that

$$
\left|N\left(t, B, H^{m}\left(Q_{\varepsilon}\left(x_{0}\right)\right)\right)-\mu_{B}\left(Q_{\varepsilon}\left(x_{0}\right)\right) t^{n / 2 m}\right| \leqq C\left(1+\varepsilon^{n-\tau} t^{(n-\tau) / 2 m}\right)
$$

for $t \geqq 0$. The same statement is valid if we replace $H^{m}$ by $H_{0}^{m}$.

Proof. Let $Q=(-1 / 2,1 / 2)^{n}$. We define the mapping $\phi$ from $Q_{\varepsilon}\left(x_{0}\right)$ onto $Q$ by

$$
y=\phi(x)=\left(x-x_{0}\right) / \varepsilon .
$$

For a function $f(x)$ defined on $Q_{\varepsilon}\left(x_{0}\right)$ we denote $f \circ \phi^{-1}(y)$ simply by $f(y)$. We consider the form

$$
\widetilde{B}[u, v]=\int_{Q} \sum_{j=1}^{n} \widetilde{p}_{j}(y) D_{j}^{m} u(y) \overline{D_{j}^{m} v(y)} d y
$$

and the operator $A$ associated with $\left\{\tilde{B}, H^{m}(Q), L_{2}(Q)\right\}$.

Let $A$ be the operator associated with $\left\{B, H^{m}(\Omega), L_{2}(\Omega)\right\}$. It is seen that

$$
A u=\varepsilon^{-2 m} A \tilde{u},
$$

from which it follows that

$$
N(t, A)=N\left(\varepsilon^{2 m} t, A\right) .
$$

On the other hand, it is easily seen that

$$
\left|\tilde{p}_{j}\right|_{0, Q} \leqq\left|p_{j}\right|_{0}, \quad\left|\tilde{p}_{j}\right|_{\tau, Q} \leqq \mathcal{E}^{\tau}\left|p_{j}\right|_{\tau} \leqq\left|p_{j}\right|_{\tau}, \quad m_{Q}\left(\tilde{p}_{j}\right) \geqq m\left(p_{j}\right) .
$$

Therefore applying Corollary 4.3 to $A$ which may depend on $\varepsilon$ and $x_{0}$, we conclude that there exists $C>0$ independent of $\varepsilon, x_{0}$ and $t$ such that

$$
\left|N(t, \tilde{A})-\mu_{\tilde{A}}(\Omega) t^{n / 2 m}\right| \leqq C\left(1+t^{(n-\tau) / 2 m}\right)
$$

for $t \geqq 0$. Replacing $t$ by $\varepsilon^{2 m} t$ in the above inequality and using (4.9) we obtain the desired result when $V=H^{m}(\Omega)$. The assertion in the case $V=H_{0}^{m}(\Omega)$ can be proved in the same way.

Q.E.D. 
For each nonnegative integer $k$, let $\left\{Q_{k, v}\right\}_{\nu=1}^{\infty}$ be a countable family of congruent and nonoverlapping open cubes of side $\varepsilon_{k}=2^{-k}$ such that $\cup_{\nu=1}^{\infty} Q_{k, \nu}=$ $\boldsymbol{R}^{n}$. In addition, each side of the cube $Q_{k, v}$ is to be parallel to one of the axes and the cubes $Q_{k, \nu}$ of the $k$ th family are to be obtained by halving the sides of each cube of the $(k-1)$ th family.

We approximate the domain $\Omega$ by unions of cubes as in [4]. We define the index set $I_{k}, J_{k}$, and the open set $\Omega_{k}, \Omega_{k}^{\prime}$ by induction on $k$ as follows:

$$
\begin{array}{ll}
I_{0}=\left\{\nu ; Q_{0, \nu} \subset \Omega\right\}, & J_{0}=\left\{\nu ; Q_{0, \nu} \cap \partial \Omega \neq \phi\right\}, \\
\Omega_{0}=\cup_{\nu \in I_{0}} Q_{0, \nu}, & \Omega_{0}^{\prime}=\Omega_{0} \cup\left(\cup_{\nu \in J_{0}} Q_{0, \nu}\right), \\
I_{k}=\left\{\nu ; Q_{k, \nu} \subset \Omega \backslash \Omega_{k-1}\right\}, & J_{k}=\left\{\nu ; Q_{k, \nu} \cap \partial \Omega \neq \phi\right\}, \\
\Omega_{k}=\cup_{l=0}^{k} \cup_{\nu \in I_{l}} Q_{l, \nu}, & \Omega_{k}^{\prime}=\Omega_{k} \cup\left(\cup_{\nu \in J_{k}} Q_{k, \nu}\right) .
\end{array}
$$

It is clear that

$$
\Omega_{k-1} \subset \Omega_{k} \subset \Omega \subset \Omega_{k}^{\prime} \subset \Omega_{k-1}^{\prime} \text {. }
$$

From Lemma 4.4 there exists $C>0$ independent of $k, \nu$ and $t$ such that

$$
\left|N\left(t, B, H^{m}\left(Q_{k, \nu}\right)\right)-\mu_{B}\left(Q_{k, \nu}\right) t^{n / 2 m}\right| \leqq C\left(1+\varepsilon_{k}^{n-\tau} t^{(n-\tau) / 2 m}\right)
$$

holds for $t \geqq 0$. (4.10) also holds if we replace $H^{m}$ by $H_{0}^{m}$.

For an index set $I$ we denote by $|I|$ the number of elements of $I$. Although we have used $|I|$ to denote the length of an interval $I$, this notation should not create any confusion. Because $Q_{k, \nu}, \nu \in I_{k}$ is contained in $\widetilde{\Gamma}_{c \varepsilon_{k}}$ with $c=2 \sqrt{n}$ and becasue $\lim \sup _{\varepsilon \rightarrow 0}\left|\widetilde{\Gamma}_{\varepsilon}\right| / \varepsilon<\infty$, there exists $C>0$ independent of $k$ such that

$$
\left|I_{k}\right| \varepsilon_{k}^{n} \leqq \sum_{\nu \in I_{k}}\left|Q_{k, \nu}\right| \leqq\left|\widetilde{\Gamma}_{c \varepsilon_{k}}\right| \leqq C \varepsilon_{k},
$$

from which it follows that

$$
\begin{gathered}
\sum_{l=0}^{k}\left|I_{l}\right| \leqq C \sum_{l=0}^{k} \varepsilon_{l}^{1-n} \leqq C \sum_{l=0}^{k} 2^{l(n-1)} \leqq C 2^{k(n-1)}, \\
\sum_{l=0}^{k}\left|I_{l}\right| \varepsilon_{l}^{n-\tau} \leqq C \sum_{l=0}^{k} \varepsilon_{l}^{1-\tau} \leqq \begin{cases}C & (0<\tau<1) \\
C k & (\tau=1) .\end{cases}
\end{gathered}
$$

For sake of simplicity we write $N\left(t, B, H^{m}\left(\Omega^{\prime}\right), L_{2}\left(\Omega^{\prime}\right)\right)$ by $N\left(t, H^{m}\left(\Omega^{\prime}\right)\right)$ and so on. The method of Dirichlet-Neumann bracketing ([4], [25]) gives

$$
\begin{aligned}
\sum_{l=0}^{k} \sum_{\nu \in I_{l}} N\left(t, H_{0}^{m}\left(Q_{l, \nu}\right)\right) & \leqq N\left(t, H_{0}^{m}(\Omega)\right) \\
& \leqq\left(\sum_{l=0}^{k} \sum_{\nu \in I_{l}}+\sum_{\nu \in J_{k}}\right) N\left(t, H^{m}\left(Q_{l, \nu}\right)\right)
\end{aligned}
$$


Hence combining (4.10)-(4.12) we have

$$
\begin{aligned}
N & \left(t, H_{0}^{m}(\Omega)\right)-\mu_{B}(\Omega) t^{n / 2 m} \\
& \geqq \sum_{l=0}^{k} \sum_{\nu \in I_{l}}\left\{N\left(t, H_{0}^{m}\left(Q_{l, \nu}\right)-\mu_{B}\left(Q_{l, \nu}\right) t^{n / 2 m}\right\}-\left\{\mu_{B}(\Omega)-\sum_{l=0}^{k} \sum_{\nu \in I_{l}} \mu_{B}\left(Q_{l, \nu}\right)\right\} t^{n / 2 m}\right. \\
& \geqq-C \sum_{l=0}^{k}\left|I_{l}\right|\left(1+\varepsilon_{l}^{n-\tau} t^{(n-\tau) / 2 m}\right)-C\left|\Gamma_{c \varepsilon_{k}}\right| t^{n / 2 m} \\
& \geqq \begin{cases}-C\left(2^{k(n-1)}+t^{(n-\tau) / 2 m}+2^{-k} t^{n / 2 m}\right) & (0<\tau<1) \\
-C\left(2^{k(n-1)}+k t^{(n-1) / 2 m}+2^{-k} t^{n / 2 m}\right) & (\tau=1)\end{cases}
\end{aligned}
$$

for $t \geqq 0$. Now we choose an integer $k$ so that $2^{k} \leqq t^{1 / 2 m}<2^{k+1}$ and obtain

$$
N\left(t, H_{0}^{m}(\Omega)\right)-\mu_{B}(\Omega) t^{n / 2 m} \geqq \begin{cases}-C\left(1+t^{(n-\tau) / 2 m}\right) & (0<\tau<1) \\ -C\left(1+t^{(n-1) / 2 m} \log t\right) & (\tau=1)\end{cases}
$$

for $t \geqq 1$. Similarly we get the estimate from above. Hence we obtain (WF1) when $0<\tau<1$ and (WF2) when $\tau=1$ for the sesquilinear form defined by (4.7) and (4.8).

This result leads us to Theorem A.(iii) through the same argument as in the end of section 4.1 .

\section{Heat Kernels}

In this section we shall prove Theorem B. We assume $2 m>n$ throughout this section.

\subsection{Resolvent kernels and heat kernels in the whole space}

Let $\mathscr{B}[u, v]$ be a symmetric sesquilinear form:

$$
\mathscr{B}[u, u]=\int_{\boldsymbol{R}^{n}} \sum_{|\alpha|,|\beta| \leq m} a_{\alpha \beta}(x) D^{\alpha} u(x) \overline{D^{\beta} v(x)} d x, \quad a_{\alpha \beta} \in \mathscr{B}^{\infty}\left(\boldsymbol{R}^{n}\right),
$$

which is strongly coercive:

$$
\mathscr{B}[u, u] \geqq \delta\|u\|_{m}^{2}, \quad \delta>0 \quad \text { for any } u \in H^{m}\left(\boldsymbol{R}^{n}\right) .
$$

From the regularity theorem of elliptic operators it follows that the positive self-adjoint operator $\mathcal{A}$ associated with $\left\{\mathscr{B}, H^{m}\left(\boldsymbol{R}^{n}\right), L_{2}\left(\boldsymbol{R}^{n}\right)\right\}$ satisfies

$$
\mathcal{A} u=\sum_{|\alpha| \leq 2 m} a_{\infty}(x) D^{\alpha} u, \quad D(\mathcal{A})=H^{2 m}\left(\boldsymbol{R}^{n}\right),
$$

where $a_{\alpha} \in \mathscr{B}^{\infty}\left(\boldsymbol{R}^{n}\right)$ is determined by (2.3). We set 


$$
\begin{aligned}
& a_{2 m}(x, \xi, \lambda)=a(x, \xi)-\lambda=\sum_{|\alpha|=2 m} a_{\alpha}(x) \xi^{\alpha}-\lambda, \\
& a_{j}(x, \xi, \lambda)=a_{j}(x, \xi)=\sum_{|\alpha|=j} a_{\alpha}(x) \xi^{\alpha} \quad(0 \leqq j \leqq 2 m-1) .
\end{aligned}
$$

Let $N$ be a positive integer and let $\lambda \in C \backslash[0, \infty)$. For $N$ and $\lambda$ we construct a parametrix $B_{N}(\lambda)$ for $\mathscr{A}-\lambda$ which satisfies

$$
(\mathcal{A}-\lambda) \cdot B_{N}(\lambda) \fallingdotseq 1, \quad B_{N}(\lambda)=\sum_{j=0}^{N-1} b_{-2 m-j}(x, D, \lambda),
$$

where $b_{-2 m-j}(x, D, \lambda)$ is the pseudo-differential operator with the symbol $b_{-2 m-j}$ $(x, \xi, \lambda)$ defined successively as follows.

$$
\begin{gathered}
a_{2 m} b_{-2 m}=1, \\
a_{2 m} b_{-2 m-j}+\sum_{l<j, l+k+|\alpha|=j} \frac{1}{\alpha !} \partial_{\xi}^{\alpha} a_{2 m-k} D_{x}^{\alpha} b_{-2 m-l}=0 \quad(j \geqq 1) .
\end{gathered}
$$

We note that $b_{-2 m-j}(x, \xi, \lambda)$ is a homogeneous function of order $-2 m-j$ in $\left(\xi, \lambda^{1 / 2 m}\right)$, that is,

$$
b_{-2 m-j}\left(x, t \xi, t^{2 m} \lambda\right)=t^{-2 m-j} b(x, \xi, \lambda), \quad t>0 .
$$

We set

$$
C_{N}(\lambda)=(\mathcal{A}-\lambda) \cdot B_{N}(\lambda)-1
$$

and have

$$
(\mathcal{A}-\lambda)^{-1}=B_{N}(\lambda)-(\mathcal{A}-\lambda)^{-1} C_{N}(\lambda) .
$$

We denote by $c_{N}(x, \xi, \lambda)$ the symbol of the pseudo-differential operator $C_{N}(\lambda)$.

The above construction of the parametrix $B_{N}(\lambda)$ is well known ([5], [6], [7], [23], [31]). In order to prove Theorem $B$ we must estimate the symbols of $B_{N}(\lambda)$ and $C_{N}(\lambda)$ more precisely so that we see how they depend on the differentiability of the coefficients $a_{\alpha}(x)$. In what follows we write $\mathscr{F}_{j, n, m, 1}$ (see section 2.3) simply by $\mathscr{F}_{j}$.

Lemma 5.1. For a multi-index $\alpha$ we have

$$
D_{x}^{\alpha} b_{-2 m-j}(x, \xi, \lambda)=\sum_{k=0}^{2 j+|\alpha|} \sum_{|\gamma|=2 m k-j} \frac{b_{j k}^{\alpha \gamma}(x) \xi^{\gamma}}{(a(x, \xi)-\lambda)^{k+1}}
$$

with the sum taken over $2 m k-j \geqq 0$ where $b_{j k}^{\alpha \gamma}(x)$ can be written

$$
b_{j k}^{\alpha \gamma}=Q_{j k}^{\alpha \gamma}[\mathcal{A}](x), \quad Q_{j k}^{\alpha \gamma} \in \mathscr{F}_{j+|\alpha|} .
$$

$Q_{j k}^{\alpha \gamma}$ is determined only by $m, n, \alpha, \gamma, j$ and $k$ and independent of the operator A. In particular we have 


$$
b_{00}^{00}(x)=1 .
$$

Proof. We prove Lemma 5.1 by induction on $j$. For sake of simplicity we write

$$
p=a(x, \xi)-\lambda
$$

For $j=0$ we have

$$
b_{-2 m}(x, \xi, \lambda)=(a(x, \xi)-\lambda)^{-1}=p^{-1} .
$$

Since

$$
D_{x_{i}} p^{-1}=-p^{-1} \cdot D_{x_{i}} p \cdot p^{-1}
$$

we obtain by induction

$$
\begin{aligned}
D_{x}^{\alpha} b_{-2 m} & =\sum C_{\alpha_{1}, \cdots, \alpha_{k}}\left(p^{-1} D_{x}^{\alpha} p\right) \cdots\left(p^{-1} D_{x}^{\alpha} p\right) \cdot p^{-1} \\
& =\sum C_{\alpha_{1}, \cdots, \alpha_{k}} \frac{D_{x}^{\alpha} p \cdots D_{x}^{\alpha} p}{(a(x, \xi)-\lambda)^{k+1}}
\end{aligned}
$$

with the sum taken over $\alpha_{1}+\cdots+\alpha_{k}=\alpha$ and $k \leqq|\alpha|$, where $C_{\alpha_{1}, \cdots, \alpha_{k}}$ is a constant. From this equality it is easily seen that the assertion for $j=0$ is valid.

Suppose that the assertion for $l$ less than $j$ is valid. Then the assertion for $j$ is seen from (5.4) and (5.7).

Hence the assertion is valid for any $j \geqq 0$.

Q.E.D.

Lemma 5.2. We have

$$
c_{N}(x, \xi, \lambda)=\sum_{j=N}^{N+2 m-1} c_{N,-j}(x, \xi, \lambda),
$$

where $c_{N,-j}(x, \xi, \lambda)$ is homogeneous of order $-j$ in $\left(\xi, \lambda^{1 / 2 m}\right)$ and satisfies

$$
c_{N,-j}(x, \xi, \lambda)=\sum_{k=0}^{2 N+2 m-2} \sum_{|\gamma|=2 m(k+1)-j} \frac{c_{N j k}^{\gamma}(x) \xi^{\gamma}}{(a(x, \xi)-\lambda)^{k+1}}
$$

with the sum taken over $2 m(k+1)-j \geqq 0$ where $c_{N j k}^{\gamma}(x)$ can be written

$$
c_{N j k}^{\gamma}=Q_{N j k}^{\gamma}[\mathcal{A}], \quad Q_{N j k}^{\gamma} \in \mathscr{F}_{j} .
$$

$Q_{N j k}^{\gamma}$ is determined only by $m, n, \gamma, N, j$ and $k$ and independent of the operator $\mathcal{A}$.

Proof. By the product formula for pseudo-differcntial operators we have

$$
c_{N}(x, \xi, \lambda)=\sum_{\alpha, k, l} \frac{1}{\alpha !} \partial_{\xi}^{\alpha} \alpha_{2 m-k} D_{x}^{\beta} b_{-2 m-l}
$$

with the sum taken over $|\alpha|+k+l \geqq N, l<N, 0 \leqq k \leqq 2 m$ and $|\alpha| \leqq 2 m-k$. Combining Lemma 5.1 and (5.8) we get Lemma 5.2.

Q.E.D. 


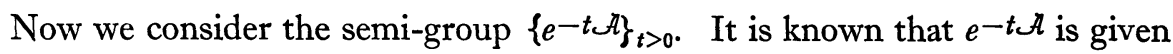
by the Laplace transform of $(\mathcal{A}-\lambda)^{-1}$ :

$$
e^{-t \mathcal{A}}=\frac{1}{2 \pi \sqrt{-1}} \int_{\Sigma}(\mathcal{A}-\lambda)^{-1} e^{-t \lambda} d \lambda,
$$

where $\Sigma$ is a path in the region $\boldsymbol{C} \backslash[0, \infty)$ which comes from $\infty e^{-\sqrt{-1} \sigma}$ and surrounds the origin clockwise and goes to $\infty e^{\nu-1} \sigma$ for some $\sigma, 0<\sigma<\pi / 2$.

Let $\mathcal{U}(t, x, y)$ be the integral kernel of $e^{-t \mathcal{A}}$. We want to evaluate $\mathcal{U}(t, x, x)$. For sake of simplicity we define some notations. Generally for an integral operator $T$ on $L_{2}(\Omega)$ we denote its integral kernel by $\mathcal{K}[T]$ :

$$
T f(x)=\int_{\mathbf{Q}} \mathcal{K}[T](x, y) f(y) d y .
$$

For a multi-index $\alpha$ and a nonnegative integer $j$ we set

$$
d_{j}^{\alpha}(x)=\int_{S} a(x, \omega)^{-(n+j) / 2 m} \omega^{\alpha} d \omega,
$$

where $S$ stands for the unit sphere in $\boldsymbol{R}^{n}$.

Lemma 5.3. $\quad B_{N}(\lambda)$ has a continuous kernel and it follows that for $t>0$

$$
\frac{1}{2 \pi \sqrt{-1}} \int_{\Sigma} \mathcal{K}\left[B_{N}(\lambda)\right](x, x) e^{-t \lambda} d \lambda=\sum_{j=0}^{N-1} b_{j}(x) t^{(j-n) / 2 m},
$$

where

$$
b_{j}(x)=\sum_{k=0}^{2 j} \sum_{|\gamma|=2 m k-j} \frac{(2 \pi)^{-n}}{2 m \cdot k !} \Gamma\left(\frac{n+|\gamma|}{2 m}\right) d_{|\gamma|}^{\gamma}(x) b_{j k}^{0 \gamma}(x)
$$

with the sum taken over $2 m k-j \geqq 0$. In particular we have

$$
b_{0}(x)=\Gamma\left(\frac{n}{2 m}+1\right) \mu_{\mathcal{A}}(x) \text {. }
$$

REMARK. $\quad b_{j k}^{0 \gamma}$ was defined in Lemma 5.1 and satisfies

$$
b_{j k}^{0 \gamma}=Q_{j k}^{0 \gamma}[\mathcal{A}], \quad Q_{j k}^{0 \gamma} \in \mathscr{F}_{j} .
$$

Proof. We consider the pseudo-differential operator $P(\lambda)$ with a symbol

$$
p(x, \xi, \lambda)=\frac{b(x) \xi^{\gamma}}{(a(x, \xi)-\lambda)^{k+1}}, \quad b \in \mathscr{B}^{\infty}\left(\boldsymbol{R}^{n}\right)
$$

with $|\gamma|-2 m(k+1)<-n$. The kernel of $P(\lambda)$ is continuous and given by

$$
\mathcal{K}[P(\lambda)](x, y)=(2 \pi)^{-n} \int_{R^{n}} e^{\sqrt{-1}(x-y) \xi} p(x, \xi, \lambda) d \xi .
$$


In particular we have

$$
\mathcal{K}[P(\lambda)](x, x)=(2 \pi)^{-n} \int_{R^{n}} p(x, \xi, \lambda) d \xi .
$$

By Cauchy's integral formula and using the polar coordinates, we have

$$
\begin{aligned}
& \frac{1}{2 \pi \sqrt{-1}} \int_{\Sigma} \mathcal{K}[P(\lambda)](x, x) e^{-t \lambda} d \lambda \\
& =(2 \pi)^{-n} \int_{R^{n}} \frac{1}{k !} t^{k} b(x) \xi^{\gamma} e^{-t a(x, \xi)} d \xi \\
& =\frac{(2 \pi)^{-n}}{2 m \cdot k !} \Gamma\left(\frac{n+|\gamma|}{2 m}\right) d_{|\gamma|}^{\gamma}(x) b(x) t^{k-(n+|\gamma|) / 2 m} .
\end{aligned}
$$

This combined with Lemma 5.1 gives (5.11).

(5.12) follows from the definition of $\mu_{\mathcal{A}}(x)$ and the equality

$$
\int_{a(x, \xi)<1} d \xi=\frac{1}{n} \int_{S} a(x, \omega)^{-n / 2 m} d \omega .
$$

Q.E.D.

In the following we denote by $C_{n, m}, \ldots$ or $c_{n, m}, \ldots$ positive constants depending only on $n, m, \cdots$.

Lemma 5.4. Let $N>n$. Then $C_{N}(\lambda)$ has a continuous kernel and there exists a constant $C_{n, m, N}>0$ such that

$$
\begin{aligned}
& \left|\mathcal{K}\left[C_{N}(\lambda)\right](x, y)\right| \\
& \quad \leqq C_{n, m, N} \sum_{j=N}^{N+2 m-1}\left(\sum_{k, \gamma}\left|d_{|\gamma|}^{0}\right|_{0}\left|c_{N j k}^{\gamma}\right|_{0}\right)|\lambda|^{(n-j) / 2 m}
\end{aligned}
$$

for $\lambda,|\lambda| \leqq 2 d(\lambda)$ with the sum taken over $k=0, \cdots, 2 N+2 m-2$ and $|\gamma|=$ $2 m(k+1)-j \geqq 0$.

Proof. Let $P(\lambda)$ be defined as in the proof of Lemma 5.3. Using (5.13) and Lemma 2.6 and changing variables we have

$$
\begin{aligned}
|\mathcal{K}[P(\lambda)](x, y)| \leqq \int_{R^{n}} \frac{(2 \pi)^{-n} 4^{k+1}|b(x)|\left|\xi^{\gamma}\right|}{(a(x, \xi)+|\lambda|)^{k+1}} d \xi \\
\leqq \frac{(2 \pi)^{-n} 4^{k+1}}{2 m \cdot k !} \Gamma\left(\frac{n+|\gamma|}{2 m}\right) \Gamma\left(k+1-\frac{n+|\gamma|}{2 m}\right) \\
\quad \times d_{|\gamma|}^{0}(x)|b(x)||\lambda|^{(n+|\gamma|) / 2 m-(k+1)} .
\end{aligned}
$$

This combined with Lemma 5.2 gives Lemma 5.4.

Q.E.D. 
We set

$$
M=\max \left\{\left|a_{\alpha \beta}\right|_{0}, 1 ;|\alpha| \leqq m,|\beta| \leqq m\right\}, \quad \delta_{1}=\min \{\delta, 1\} .
$$

Lemma 5.5. There exist $C_{n, m}>0$ and $c_{n, m}>0$ such that

$$
\begin{aligned}
& \left|\mathcal{K}\left[(\mathcal{A}-\lambda)^{-1}\right](x, y)\right| \\
& \quad \leqq C_{n, m} \delta_{1}^{-1}|\lambda|^{n / 2 m-1} \exp \left(-c_{n, m} \delta_{1} M^{-1}|\lambda|^{1 / 2 m}|x-y|\right)
\end{aligned}
$$

for $\lambda, 1 \leqq|\lambda| \leqq 2 d(\lambda)$.

Lemma 5.5 is essentially due to Tsujimoto [32], who proved it when $\mathcal{A}$ is an operator on a bounded domain in $\boldsymbol{R}^{n}$. We must prove it when $\mathcal{A}$ is an operator on the whole space $\boldsymbol{R}^{n}$. Furthermore we need to estimate $\mathcal{K}\left[(\mathcal{A}-\lambda)^{-1}\right]$ $(x, y)$ paying attention to the constants $M$ and $\delta_{1}$. Lemma 5.5 will be proved in Appendix.

Lemma 5.6. Let $N>n$. We have for $0<t \leqq 1$

$$
\begin{aligned}
& \left|\frac{1}{2 \pi \sqrt{-1}} \int_{\Sigma} e^{-t \lambda} \mathcal{K}\left[(\mathcal{A}-\lambda)^{-1} C_{N}(\lambda)\right](x, y) d \lambda\right| \\
& \quad \leqq C_{n, m, N} \delta_{1}^{-(n+1)} M^{n} \sum_{j=N}^{N+2 m-1}\left(\sum_{k, \gamma}\left|d_{|\gamma|}^{0}\right|_{0}\left|c_{N j k}^{\gamma}\right|_{0}\right) t^{(j-n) / 2 m}
\end{aligned}
$$

with the sum taken over $k=0, \cdots, 2 N+2 m-2$ and $|\gamma|=2 m(k+1)-j \geqq 0$.

Proof. Let $1 \leqq|\lambda| \leqq 2 d(\lambda)$. From Lemma 5.5 it follows that

$$
\int_{R^{n}}\left|\mathcal{K}\left[(\mathcal{A}-\lambda)^{-1}\right](x, y)\right| d y \leqq C_{n, m} \delta_{1}^{-(n+1)} M^{n}|\lambda|^{-1}
$$

This combined with Lemma 5.4 gives

$$
\begin{aligned}
& \left|\mathcal{K}\left[(\mathcal{A}-\lambda)^{-1} C_{N}(\lambda)\right](x, x)\right| \\
& \quad \leqq \int_{R^{n}}\left|\mathcal{K}\left[(\mathcal{A}-\lambda)^{-1}\right](x, y) \mathcal{K}\left[C_{N}(\lambda)\right](y, x)\right| d y \\
& \quad \leqq C_{n, m, N} \delta_{1}^{-(n+1)} M^{n} \sum_{j=N}^{\pi+2 m+1}\left(\sum_{k, \gamma}\left|d_{|\gamma|}^{0}\right|_{0}\left|c_{N j k}^{\gamma}\right|_{0}\right)|\lambda|^{(n-j) / 2 m-1} .
\end{aligned}
$$

Let $q(\lambda)$ be a function defined on $\{\lambda ; 1 \leqq|\lambda| \leqq 2 d(\lambda)\}$ which satisfies

$$
|q(\lambda)| \leqq|\lambda|^{\rho-1}
$$

for some $\rho \in \boldsymbol{R}$. For a fixed $t, 0<t \leqq 1$ we define the path $\Sigma_{t}$ in $\{\lambda ; 1 \leqq|\lambda| \leqq$ $2 d(\lambda)\}$ by

$$
\begin{aligned}
& \Sigma_{t}=\Gamma_{+} \cup \Gamma_{0} \cup \Gamma_{-}, \\
& \Gamma_{ \pm}=\left\{\lambda \in C ;|\lambda| \geqq t^{-1}, \operatorname{Im} \lambda= \pm \operatorname{Re} \lambda\right\} \\
& \Gamma_{0}=\left\{\lambda \in C ; \lambda=-t^{-1} e^{i \sigma},-\frac{3}{4} \pi \leqq \sigma \leqq \frac{3}{4} \pi\right\}
\end{aligned}
$$


Then we have

$$
\begin{aligned}
& \left|\frac{1}{2 \pi \sqrt{-1}} \int_{\Sigma_{t}} e^{-t \lambda} q(\lambda) d \lambda\right| \\
& \quad \leqq 2 \cdot \frac{1}{2 \pi} \int_{t^{-1}}^{\infty} e^{-t s / \sqrt{2}} s^{\rho-1} d s+\frac{1}{2 \pi} \int_{-3 \pi / 4}^{3 \pi / 4} e\left(t^{-1}\right)^{\rho-1} t^{-1} d \sigma \\
& \quad \leqq\left(\pi^{-1} 2^{\rho / 2} \int_{1 / 2}^{\infty} s^{\rho-1} e^{-s} d s+e\right) t^{-\rho} .
\end{aligned}
$$

Since $\mathcal{K}\left[(\mathcal{A}-\lambda)^{-1} C_{N}(\lambda)\right](x, y)$ is analytic in $\lambda$ we may change the path $\Sigma$ into $\Sigma_{t}$. Hence from (5.14) and (5.15) we get Lemma 5.6.

Q.E.D.

Combining Lemma 5.3, Lemma 5.6, (5.6) and (5.9) we conclude the following.

Lemma 5.7. Let $N>n$. We have for $0<t \leqq 1$

$$
\begin{aligned}
& \left|\mathcal{U}(x, x ; t)-\sum_{j=0}^{N-1} b_{j}(x) t^{(j-n) / 2 m}\right| \\
& \quad \leqq C_{n, m, N} \delta_{1}^{-(n+1)} M^{n} \sum_{j=N}^{N+2 m-1}\left(\sum_{k, \gamma}\left|d_{|\gamma|}^{0}\right|_{0}\left|c_{N j k}^{\gamma}\right|_{0}\right) t^{(j-n) / 2 m}
\end{aligned}
$$

with the sum taken over $k=0, \cdots, 2 N+2 m-2$ and $|\gamma|=2 m(k+1)-j \geqq 0$.

\subsection{Traces of heat kernels in $\Omega$}

Let $\Omega$ be a bounded domain with the restricted cone property. Let $V$ be a closed subspace of $H^{m}(\Omega)$ containing $H_{0}^{m}(\Omega)$. Let $\mathscr{B}[u, v]$ be a symmetric sesquilinear form satisfying (5.1) and (5.2). Furthermore we assume that the restriction of $\mathscr{B}$ to $\Omega$, which is denoted by $B$ :

$$
B[u, v]=\int_{\alpha} \sum_{|\alpha|,|\beta| \leqq m} a_{\alpha \beta}(x) D^{\alpha} u(x) \overline{D^{\beta} v(x)} d x
$$

is also strongly coercive on $V$ :

$$
B[u, u] \geqq \delta\|u\|_{m}^{2} \quad \text { for any } u \in V,
$$

where $\delta$ is the same constant as in (5.2). Let $\mathcal{A}$ (resp. $A$ ) be the operator associated with the triple $\left\{\mathscr{B}, H^{m}\left(\boldsymbol{R}^{n}\right), L_{2}\left(\boldsymbol{R}^{n}\right)\right\}$ (resp. $\left.\left\{B, V, L_{2}(\Omega)\right\}\right)$. We denote by $\mathcal{U}(t, x, y)$ (resp. $U(t, x, y))$ the heat kernel of $\mathcal{A}$ (resp. $A$ ) and put

$$
U(t)=\int_{\Omega} U(t, x, x) d x .
$$

In order to estimate $U(t)$ we prepare two lemmas.

Lemma 5.8 ([14]).

(i) There exists $C_{n, m, 0}>0$ such that 


$$
\left|\mathcal{K}\left[(A-\lambda)^{-1}\right](x, y)\right| \leqq C_{n, m, 0} \delta_{1}^{-1} M \frac{|\lambda|^{n / 2 m}}{d(\lambda)}
$$

for $x, y \in \Omega$ and $\lambda \in C \backslash[0, \infty)$.

(i)' (i) is also valid if we replace $A$ and $\Omega$ by $\mathcal{A}$ and $\boldsymbol{R}^{n}$.

(ii) Let $0 \leqq \theta \leqq 1$. There exists $C_{n, m, \theta, \Omega}>0$ such that

$$
\begin{array}{r}
\left|\mathcal{K}\left[(A-\lambda)^{-1}\right](x, x)-\mathcal{K}\left[(\mathcal{A}-\lambda)^{-1}\right](x, x)\right| \\
\leqq C_{m, n, \theta, 0} \delta_{1}^{-2} M^{2} \frac{|\lambda|^{n / 2 m}}{d(\lambda)}\left(\frac{|\lambda|^{1-1 / 2 m}}{\delta(x) d(\lambda)}\right)^{\theta}
\end{array}
$$

for $x, y \in \Omega$ and $\lambda \in C \backslash[0, \infty),|\lambda| \geqq 1$.

Proof. From Lemma 2.6 we have

$$
\begin{aligned}
|B[u, u]-\lambda(u, u)| & \geqq \frac{d(\lambda)}{2|\lambda|}\left(B[u, u]+|\lambda|\|u\|_{0}^{2}\right) \\
& \geqq \frac{\delta_{1} d(\lambda)}{4|\lambda|}\left(\|u\|_{m}+|\lambda|^{1 / 2}\|u\|_{0}\right)^{2} .
\end{aligned}
$$

To prove Lemma 5.8 we have only to follow the proof of Maruo and Tanabe [14, Lemma 3.1] (resp. [14, Lemmas 4.1, 4.2]) for (i) (resp. (ii)), paying the attention to the constants $\delta_{1}$ and $M$, and using (5.17).

Q.E.D.

Lemma 5.9. There exists a constant $C_{\Omega}>0$ such that the following inequalities hold.

(i) $\left|\Gamma_{\varepsilon}\right| \leqq C_{\Omega} \varepsilon$ for $\varepsilon>0$.

(ii) $\int_{\Omega} \delta(x)^{-\theta} d x<\infty$ for $0<\theta<1$.

(iii) $\int_{\delta(x)>\varepsilon} \delta(x)^{-1} d x \leqq C_{\mathbf{Q}}\left(\log ^{+} \varepsilon^{-1}+1\right)$ for $\varepsilon>0$,

where $\log ^{+} t=\max \{\log t, 0\}$.

Proof. Since $\Omega$ possesses the restricted cone property, (i) holds (see [16]). (ii) and (iii) follow from (i). The details are found in [21].

Proposition 5.10. Let $0<\theta \leqq 1$. We have for $0<t \leqq 1$

$$
\begin{aligned}
\mid U(t) & -\Gamma\left(\frac{n}{2 m}+1\right) \mu_{A}(\Omega) t^{-n / 2 m} \mid \\
& \leqq C_{n, m, \theta, \Omega} \delta_{1}^{-(n+1)} M^{n+1}\left(\sum_{j=1}^{n+2 m} \tilde{b}_{j} t^{(j-n) / 2 m}+J_{\theta}(t)\right),
\end{aligned}
$$

where 


$$
\begin{aligned}
\tilde{b}_{j} & = \begin{cases}\left|b_{j}\right|_{0} & (1 \leqq j \leqq n) \\
\sum_{k, \gamma}\left|d_{|\gamma|}^{0}\right|_{0}\left|c_{(n+1) j k}^{\gamma}\right|_{0} & (n+1 \leqq j \leqq n+2 m),\end{cases} \\
J_{\theta}(t) & = \begin{cases}t^{(\theta-n) / 2 m} & (0<\theta<1) \\
t^{(1-n) / 2 m}\left(\log t^{-1}+1\right) & (\theta=1) .\end{cases}
\end{aligned}
$$

Here the sum $\sum_{k, \gamma}$ is taken over $k=0, \cdots, 2 n+2 m$ and $|\gamma|=2 m(k+1)-j \geqq 0$.

Proof. Let $0<t \leqq 1$. Using Lemma 5.8.(ii) and (5.15) we get the estimate for the difference of heat kernels:

$$
|\mathcal{U}(t, x, x)-U(t, x, x)| \leqq C_{n, m, \theta, \Omega} \delta_{1}^{-2} M^{2} \delta(x)^{-\theta} t^{(\theta-x) / 2 m}
$$

for $0<\theta \leqq 1$. Integrating (5.16) with $N=n+1$ and (5.18) on $\Omega$ and using Lemma 5.9.(ii) we obtain the desired result for $0<\theta<1$.

It remains to prove Proposition 5.10 for $\theta=1$. From (5.18) and Lemma 5.9.(iii) we have

$$
\begin{aligned}
& \left|\int_{\delta(x) \geqq t^{1 / 2 m}}\{\bigcup(t, x, x)-U(t, x, x)\} d x\right| \\
& \leqq C_{n, m, 0} \delta_{1}^{-2} M^{2} t^{(1-n) / 2 m}\left(\log ^{+} t^{-1}+1\right) .
\end{aligned}
$$

Using Lemma 5.8.(i), (i)' and (5.15) we have

$$
|U(t, x, x)-U(t, x, x)| \leqq C_{n, m, 0} \delta_{1}^{-1} M t^{-n / 2 m}
$$

This combined with Lemma 5.9.(i) gives

$$
\left|\int_{\delta(x) \leqq t^{1 / 2 m}}\{\bigcup(t, x, x)-U(t, x, x)\} d x\right| \leqq C_{n, m, \delta} \delta_{1}^{-1} M t^{(1-n) / 2 m} .
$$

Integrating (5.16) with $N=n+1$ on $\Omega$ and combining (5.19) and (5.20) we obtain the desired result for $\theta=1$.

Q.E.D.

\subsection{Heat kernels with Hölder continuous coefficients}

Now we shall prove Theorem B. Let a variational triple $\left\{B, V, L_{2}(\Omega)\right\}$ satisfy (1.1)-(1.4) with $0<\tau \leqq 1$. We may regard $a_{\alpha \beta}$ as an element of $\mathscr{B}^{\tau}\left(\boldsymbol{R}^{n}\right)$ (see $[29, \mathrm{p} 174])$. For $\varepsilon>0$ we set

$$
a_{\alpha \beta}^{\varepsilon}=\varphi_{\mathrm{e}} * a_{\alpha \beta} \quad(|\alpha|=|\beta|=m),
$$

where $\varphi_{\varepsilon}$ is the function defined in Lemma 2.4. We define symmetric forms

$$
\begin{aligned}
& B_{\varepsilon}[u, v]=\int_{\alpha} \sum_{|\alpha|=|\beta|=m} a_{\alpha \beta}^{\varepsilon} D^{\alpha} u \overline{D^{\beta} v} d x+C_{0}(u, v)_{L_{2}(\Omega)}, \\
& \mathscr{B}_{\varepsilon}[u, v]=\int_{R^{n}} \sum_{|\alpha|=\mid \beta=m} a_{\alpha \beta}^{\varepsilon} D^{\alpha} u \overline{D^{\beta} v} d x+C_{0}(u, v)_{L_{2}\left(R^{n}\right)},
\end{aligned}
$$


where $C_{0}>0$ is a constant. Replacing the values of $a_{\alpha \beta}$ outside $\Omega$ if necessary, taking $C_{0}>0$ sufficiently large, and using (1.3), Gårding's inequality and so on, we may assume that there exists $\varepsilon_{0}, 0<\varepsilon_{0}<1$ such that

$$
\begin{gathered}
B_{\varepsilon}[u, u] \geqq \frac{\delta}{2}\|u\|_{m}^{2} \quad \text { for } 0<\varepsilon<\varepsilon_{0}, u \in V, \\
\mathcal{B}_{\varepsilon}[u, u] \geqq \frac{\delta}{2}\|u\|_{m}^{2} \quad \text { for } 0<\varepsilon<\varepsilon_{0}, u \in H^{m}\left(\boldsymbol{R}^{n}\right) .
\end{gathered}
$$

We denote the trace of the heat kernel of the operator associated with $\left\{B_{\mathfrak{z}}, V\right.$, $\left.L_{2}(\Omega)\right\}$ by $U_{\mathrm{z}}(t)$.

Lemma 5.11. Let $0<\theta \leqq 1$. There exists $C>0$ independent of $\varepsilon$ and $t$ such that

$$
\left|U_{\mathrm{z}}(t)-\Gamma\left(\frac{n}{2 m}+1\right) \mu_{A_{\mathrm{g}}}(\Omega) t^{-n / 2 m}\right| \leqq C \sum_{j=1}^{n+2 m} \varepsilon^{\tau-j} t^{(j-n) / 2 m}+C J_{\theta}(t)
$$

holds for $0 \leqq t \leqq 1$, where $J_{\theta}(t)$ was defined in Proposition 5.10 .

Proof. From (5.22), Lemma 2.4 and the inverse of Gårding's inequality it follows that the constants $\delta_{1}^{-1}, M$ and $\left|d_{|y|}^{0}\right|_{0}$ can be evaluated from above by positive constants independent of $\varepsilon$. Let $\tilde{b}_{j, \mathrm{e}}$ stand for the constant $\tilde{b}_{j}$ corresponding to the forms $B_{\varepsilon}$ and $\mathscr{B}_{\mathrm{q}}$ in Proposition 5.10. Using Lemmas 5.2, 5.3 and Lemma 2.5.(v) we have

$$
\left|\tilde{b}_{j, \varepsilon}\right| \leqq C \varepsilon^{\tau-j},
$$

where $C>0$ is independent of $\varepsilon$. Hence applying Proposition 5.10 to the forms $B_{\mathrm{z}}$ and $\mathscr{B}_{\mathrm{q}}$ we obtain the desired result.

Q.E.D.

From the interpolation inequality, Lemma 2.4 and (5.21) we have

$$
\begin{aligned}
\left|B_{\varepsilon}[u, u]-B[u, u]\right| & \leqq C \varepsilon^{\tau}\|u\|_{m}^{2}+C\|u\|_{m}\|u\|_{m-1} \\
& \leqq C\left(\varepsilon^{\tau}\|u\|_{m}^{2}+\varepsilon^{-2 m+1}\|u\|_{0}^{2}\right) \\
& \leqq C \varepsilon^{\top} B_{\varepsilon}[u, u\rfloor+C \varepsilon^{-2 m+1}\|u\|_{0}^{2},
\end{aligned}
$$

from which it follows that

$$
\begin{aligned}
& B[u, u] \geqq\left(1-C \varepsilon^{\tau}\right) B_{\varepsilon}[u, u]-C \varepsilon^{-2 m+1}\|u\|_{0}^{2}, \\
& B[u, u] \leqq\left(1+C \varepsilon^{\tau}\right) B_{\varepsilon}[u, u]+C \varepsilon^{-2 m+1}\|u\|_{0}^{2} .
\end{aligned}
$$

Noting that $1-C \varepsilon^{\tau} \geqq 1 / 2$ if $\varepsilon_{0}$ is sufficiently small, and using Lemma 2.1 we have 


$$
\begin{aligned}
& N(t, B, V) \leqq N\left(\left(1-C \varepsilon^{\tau}\right)^{-1}\left(t+C \varepsilon^{-2 m+1}\right), B_{\varepsilon}, V\right), \\
& N(t, B, V) \geqq N\left(\left(1+C \varepsilon^{\tau}\right)^{-1}\left(t-C \varepsilon^{-2 m+1}\right), B_{\varepsilon}, V\right) .
\end{aligned}
$$

We write $N(t, B, V)$ or $N\left(t, B_{\varepsilon}, V\right)$ simply by $N(t)$ or $N_{\varepsilon}(t)$ respectively. From Lemma 2.1, (5.23) and the relation between $U(t)$ and $N(t)$ we have

$$
\begin{aligned}
U(t) & =\int_{-\infty}^{\infty} e^{-t s} d N(s)=\int_{-\infty}^{\infty} t e^{-t s} N(s) d s \\
& \leqq \int_{-\infty}^{\infty} t e^{-t s} N_{\mathrm{\varepsilon}}\left(\left(1-C \varepsilon^{\tau}\right)^{-1}\left(s+C \varepsilon^{-2 m+1}\right)\right) d s \\
& =\int_{-\infty}^{\infty}\left(1-C \varepsilon^{\tau}\right) t \exp \left(-\left(1-C \varepsilon^{\tau}\right) t s+C \varepsilon^{-2 m+1} t\right) N_{\mathrm{\varepsilon}}(s) d s \\
& =U_{\varepsilon}\left(\left(1-C \varepsilon^{\tau}\right) t\right) \exp \left(C \varepsilon^{-2 m+1} t\right) .
\end{aligned}
$$

This combined with Lemma 5.11 gives

$$
\begin{aligned}
& \exp \left(-C \varepsilon^{-2 m+1} t\right) U(t) \\
& \leqq \Gamma\left(\frac{n}{2 m}+1\right) \mu_{A_{\varepsilon}}(\Omega)\left\{\left(1-C \varepsilon^{\tau}\right) t\right\}^{-n / 2 m} \\
& \quad+C \sum_{j=1}^{n+2 m} \varepsilon^{\tau-j}\left\{\left(1-C \varepsilon^{\tau}\right) t\right\}^{(j-n) / 2 m}+C J_{\theta}\left(\left(1-C \varepsilon^{\tau}\right) t\right) \\
& \leqq \Gamma\left(\frac{n}{2 m}+1\right) \mu_{A}(\Omega) t^{-n / 2 m}+C \varepsilon^{\tau} t^{-n / 2 m} \\
& \quad+C \sum_{j=1}^{n+2 m} \varepsilon^{\tau-j} t^{(j-n) / 2 m}+C J_{\theta}\left(\frac{t}{2}\right) \quad \text { for } 0<t \leqq 1
\end{aligned}
$$

Now we take $t_{0}$ so that $t_{0}^{1 / 2 m}=\varepsilon_{0}$. Putting $\theta=\tau$ and $\varepsilon=t^{1 / 2 m}$ for $0<t<t_{0}$, and noting

$$
\exp \left(C \varepsilon^{-2 m+1} t\right)=1+O\left(t^{1 / 2 m}\right)
$$

we obtain

$$
U(t) \leqq \Gamma\left(\frac{n}{2 m}+1\right) \mu_{A}(\Omega) t^{-n / 2 m}+ \begin{cases}C t^{(\tau-n) / 2 m} & (0<\tau<1) \\ C t^{(1-n) / 2 m} \log t^{-1} & (\tau=1)\end{cases}
$$

for $0<t<t_{0}$. Similarly we get the estimate for $L^{\top}(t)$ from below. Thus we complete the proof of Theorem $B$.

\section{Appendix}

We prove Lemma 5.5. Let $\mathscr{B}[u, v]$ be a sesquilinear form satisfying (5.1) and (5.2). For $\eta=\left(\eta_{1}, \cdots, \eta_{n}\right) \in C^{n}$ we set

$$
\mathscr{B}_{\eta}[u, v]=\int_{R^{n}|\alpha|,|\beta| \leqq m} a_{\alpha \beta}(x)(D+i \eta)^{\alpha} u(x) \overline{(D-i \bar{\eta})^{\beta} v(x)} d x,
$$


where $i$ stands for the imaginary unit $\sqrt{-1}$. This notation should not occur any confusion. Let $\mathscr{A}_{\eta}$ be the operator associated with $\left\{\mathscr{B}_{\eta}, H^{m}\left(\boldsymbol{R}^{n}\right)\right.$, $\left.L\left({ }_{2} \boldsymbol{R}^{n}\right)\right\}$. At first we want to evaluate $\mathcal{K}\left[\left(\mathcal{A}_{\eta}-\lambda\right)^{-1}\right](x, y)$. To this end we have only to follow Tsujimoto's proof ([32, Lemma 5.1]), paying attention to the constants $\delta_{1}$ and $M$ as follows.

Let $1 \leqq|\lambda| \leqq 2 d(\lambda)$. The same inequality as (5.17) gives

$$
|\mathscr{B}[u, u]-\lambda(u, u)| \geqq \frac{\delta_{1}}{8}\left(\|u\|_{m}+|\lambda|^{1 / 2}|| u \|_{0}\right)^{2} .
$$

Using the interpolation inequality we have

$$
\begin{aligned}
& \left|\mathscr{B}_{\eta}[u, u]-\mathscr{B}[u, u]\right| \\
& \quad \leqq C_{n, m} M \sum_{0<k+l \leqq 2 m}|\eta|^{k+l}|\lambda|^{-(k+l) / 2 m}\left(\|u\|_{m}+|\lambda|^{1 / 2}|| u \|_{0}\right)^{2} \\
& \quad \leqq C_{n, m} M|\eta||\lambda|^{-1 / 2 m}\left(\|u\|_{m}+|\lambda|^{1 / 2}\|u\|_{0}\right)^{2}
\end{aligned}
$$

if $|\eta||\lambda|^{-1 / 2 m} \leqq 1$. Hence from (A-1) and (A-2) we have

$$
\left|\mathscr{B}_{\eta}[u, u]-\lambda(u, u)\right| \geqq \frac{\delta_{1}}{16}\left(\|u\|_{m}+|\lambda|^{1 / 2}\|u\|_{0}\right)^{2},
$$

if $|\eta||\lambda|^{-1 / 2 m} \leqq C_{n, m} \delta_{1} M^{-1}$. We put

$$
u=\left(\mathcal{A}_{\eta}-\lambda\right)^{-1} f
$$

and have

$$
\left|\mathscr{B}_{\eta}[u, u]-\lambda(u, u)\right|=|(f, u)| \leqq\left\{\begin{array}{l}
\|f\|\left\|_{0}\right\| u \|_{0} \\
\|f\|_{-m}\|u\|_{m}
\end{array} .\right.
$$

Here $\|f\|_{-m}$ stands for the norm of the Sobolev space $H^{-m}\left(\boldsymbol{R}^{n}\right)$. From (A-3), (A-4) and the integral kernel theorem ([14, Lemma 3.2]) we obtain

$$
\left|\mathcal{K}\left[\left(\mathcal{A}_{\eta}-\lambda\right)^{-1}\right](x, y)\right| \leqq C_{n, m} \frac{16}{\delta_{1}}|\lambda|^{n / 2 m-1},
$$

if $|\eta||\lambda|^{-1 / 2 m} \leqq C_{n, m} \delta_{1} M^{-1}$.

If we can show that

$$
\mathcal{K}\left[(\mathcal{A}-\lambda)^{-1}\right](x, y)=e^{-\eta(x-y)} \mathcal{K}\left[\left(\mathcal{A}_{\eta}-\lambda\right)^{-1}\right](x, y)
$$

holds, then we obtain Lemma 5.5 by putting

$$
\eta=-C_{n, m} \frac{\delta_{1}}{M}|\lambda|^{1 / 2 m} \cdot \frac{(x-y)}{|x-y|}
$$

in (A-5). Since it does not always follow that

$$
u \in H^{m}\left(\boldsymbol{R}^{n}\right) \text { implies } e^{x \eta} u \in H^{m}\left(\boldsymbol{R}^{n}\right),
$$


it is not so easy to show (A-5) as in [32].

We will prove (A-5).

Step 1. Suppose $\eta \in(i \boldsymbol{R})^{n}$. Since $(\mathrm{A}-6)$ holds in this case, it is seen that $(\mathcal{A}-\lambda)^{-1} f=e^{-x^{\eta}}\left(\mathcal{A}_{\eta}-\lambda\right) e^{x^{\eta}} f$, from which (A-5) follows.

Step 2. We show the differentiability of $\mathcal{K}\left[\left(\mathcal{A}_{\eta}-\lambda\right)^{-1}\right](x, y)$ in $\eta$. Let $f \in L_{2}\left(\boldsymbol{R}^{n}\right), h \in \boldsymbol{C}$ with $h \neq 0,|h|<1$. Put

$$
v_{h}=\left(\mathscr{A}_{\eta+h e_{j}}-\lambda\right)^{-1} f-\left(\mathscr{A}_{\eta}-\lambda\right)^{-1} f,
$$

where $|\eta| \leqq C_{n, m} \delta_{1} M^{-1}|\lambda|^{1 / 2 m}$ and $\left|\eta+e_{j}\right| \leqq C_{n, m} \delta_{1} M^{-1}|\lambda|^{1 / 2 m}$. For any $v \in$ $H^{m}\left(\boldsymbol{R}^{n}\right)$ we have

$$
\begin{aligned}
& \mathscr{B}_{\eta}\left[v_{h}, v\right]-\lambda\left(v_{h}, v\right) \\
& =\mathscr{B}_{\eta+h e_{j}}\left[\left(\mathscr{A}_{\eta+h e j}-\lambda\right)^{-1} f, v\right]-\lambda\left(\left(\mathscr{A}_{\eta+h e j}-\lambda\right)^{-1} f, v\right) \\
& -\mathscr{B}_{\eta}\left[\left(\mathcal{A}_{\eta}-\lambda\right)^{-1} f, v\right]+\lambda\left(\left(\mathcal{A}_{\eta}-\lambda\right)^{-1} f, v\right) \\
& +\left(\mathscr{B}_{\eta}-\mathscr{B}_{\eta+h e j}\right)\left[\left(\mathcal{A}_{\eta+h e j}-\lambda\right)^{-1} f, v\right] \\
& (\mathrm{A}-7) \quad=\left(\mathscr{B}_{\eta}-\mathscr{B}_{\eta+h e_{j}}\right)\left[\left(\mathcal{A}_{\eta+h e_{j}}-\lambda\right)^{-1} f, v\right] \\
& =\int_{R^{n}} \sum_{\alpha, \beta} a_{\alpha \beta}(x)\left\{(D+i \eta)^{\alpha}\left(\mathcal{A}_{\eta+h e_{j}}-\lambda\right)^{-1} f \cdot \overline{(D-i \bar{\eta})^{\beta} v}\right. \\
& -\left(D+i\left(\eta+h e_{j}\right)\right)^{\alpha}\left(\mathscr{A}_{\eta+h e_{j}}-\lambda\right)^{-1} f \cdot \overline{\left(D-i\left(\bar{\eta}+\bar{h} e_{j}\right)\right)^{\beta_{\eta}} v} d x \\
& =h J_{\eta}\left[\left(\mathcal{A}_{\eta+h e j}-\lambda\right)^{-1} f, v\right]+I_{\eta}(h)\left[\left(\mathcal{A}_{\eta+h e j}-\lambda\right)^{-1} f, v\right] \text {, }
\end{aligned}
$$

where $J_{\eta}[u, v]$ and $I_{\eta}(h)[u, v]$ are sesquilinear forms satisfying the following.

$$
\begin{aligned}
& J_{\eta}[u, v]=\int_{R^{n}} \sum_{\alpha, \beta} a_{\alpha \beta}\left\{\left(D+i \eta_{\eta}\right)^{\alpha} u \cdot \overline{i \beta_{j}(D-i \bar{\eta})^{\beta-e_{j}}}\right. \\
& -i \alpha_{j}(D+i \eta)^{\alpha-e_{j} u \cdot \overline{\left.(D-i \bar{\eta})^{\beta} \boldsymbol{}\right\}}} d x, \\
& \left|J_{\eta}[u, v]\right| \leqq C\left(1+|\eta|^{2 m}\right)\|u\|_{m} \mid\|v\|_{m}, \\
& \left|I_{\eta}(h)[u, v]\right| \leqq C|h|^{2}\left(1+|\eta|^{2 m}\right)|| u\left\|_{m}\right\| v \|_{m} .
\end{aligned}
$$

Here and in what follows we denote by $C$ positive constants depending only on $n, m$ and $\sup \left|a_{\alpha \beta}(x)\right|$. Putting $v=v_{h}$ in (A-7) and using (A-3), (A-8) and (A-9) we get

$$
\left\|v_{h}\right\|_{m} \leqq C|h|\left(1+|\eta|^{2 m}\right)\left\|\left(\mathscr{A}_{\eta+h e_{j}}-\lambda\right)^{-1} f\right\|_{m} .
$$

On the other hand, from Lax-Milgram's theorem there exists $w \in H^{m}\left(\boldsymbol{R}^{n}\right)$ such that

$$
\mathscr{B}_{\eta}[w, v]-\lambda(w, v)=J_{\eta}\left[\left(\mathscr{A}_{\eta}-\lambda\right)^{-1} f, v\right]
$$

Putting

$$
u_{h}=\frac{1}{h} v_{h}-w,
$$


and combining (A-7) and (A-11) we obtain

$$
\mathscr{B}_{\eta}\left[u_{h}, u_{h}\right]-\lambda\left(u_{h}, u_{h}\right)=J_{\eta}\left[v_{h}, u_{h}\right]+\frac{1}{h} I_{\eta}(h)\left[\left(\mathcal{A}_{\eta+h e_{j}}-\lambda\right)^{-1} f, u_{h}\right]
$$

This combined with (A-3), (A-8)-(A-10) gives

$$
\begin{aligned}
\left\|u_{h}\right\|_{m}+|\lambda|^{1 / 2}\left\|u_{h}\right\|_{0} & \leqq C|h|\left(1+|\eta|^{2 m}\right)^{2}\left\|\left(A_{\eta+h e_{j}}-\lambda\right)^{-1} f\right\|_{m} \\
& \leqq\left\{\begin{array}{l}
C|h|\left(1+|\eta|^{2 m}\right)^{2}|\lambda|^{-1 / 2}\|f\|_{0} \\
C|h|\left(1+|\eta|^{2 m}\right)^{2}\|f\|_{-m} .
\end{array}\right.
\end{aligned}
$$

From the above inequality and the integral kernel theorem we conclude that $\mathcal{K}\left[\left(\mathcal{A}_{\eta}-\lambda\right)^{-1}\right](x, y)$ is holomorphic in $\eta$.

Combining step 1 and step 2 and using the uniqueness theorem we obtain (A-5). Thus we complete the proof of Lemma 5.5.

Acknowledgement. The author wishes to thank Professor Toshihusa Kimura for guidance and encouragement.

\section{References}

[1] S. Agmon: Lectures on Elliptic Boundary Value Problems, Van Nostrand Mathematical Studies, Princeton, 1965.

[2] R. Beals: Asymptotic behavior of the Green's function and spectral function of an elliptic operator, J. Funct. Anal., 5 (1970), 484-503.

[3] J. Brüning: Zur abschatzung der spektral-function elliptisher operatoren, Math. Z., 137 (1974), 75-85.

[4] R. Courant and D. Hilbert: Methods of Mathematical Physics, Interscience, New York, 1953.

[5] D. Fujiwara: On the asymptotic behavior of the Green operators for elliptic boundary problems and the pure imaginary powers of some second order operators, J. Math. Soc. Japan, 21 (1969), 481-522.

[6] P.B. Gilkey: Invariance Theory, The Heat Kernel Equation, and the AtiyahSinger Index Theorem, Publish or Perish, Wilmington, 1984.

[7] P. Geriner: An asymptotic expansion for the heat equation, Arch. Rat. Mech. Anal. 41 (1971), 163-218.

[8] P. Hratman: Ordinary Differential Equations, John Wiley, New York-London, 1964.

[9] E. Hille: Lectures in Ordinary Differential Equations, Addison-Wesley, Massachusetts, 1969.

[10] V. Ivrii: Precise Spectral Asymptotics for Elliptic Operators Acting in Fiberings over Manifolds with Boundary, Lecture Notes in Math., Vol. 1100 SpringerVerlag, Berlin, 1984.

[11] H. Kumano-go: Pseudo-differential Operators, MIT, Massachusetts, 1980.

[12] M.L. Lapidus: Fractal drum, inverse spectral problems for elliptic operators and 
a partial resolution of the Weyl-Berry conjecture, to appear in "Trans. Amer. Math. Soc.".

[13] B.M. Levitan and I.S. Sargsgan: Introduction to Spectral Theory. Self-adjoint Ordinary Differential Operators, Amer. Math. Soc., Transaction of Math. Monographs (39), 1975.

[14] K. Maruo and H. Tanabe: On the asymptotic distribution of eigenvalues of operators associated with strongly elliptic sesquilinear forms, Osaka J. Math., 8 (1971), 323-345.

[15] K. Maruo: Asymptotic distribution of eigenvalues of non-symmetric operators associated with strongly elliptic sesquilinear forms, Osaka J. Math., 9 (1972), 547560.

[16] G. Metivier: Etude asymptotique des valeurs propres et de la fonction spectrale de problemes aux limites, These de Doctorat d'Etat, Mathematiques, Universite de Nice, France, 1976.

[17] G. Métivier: Comportement asymptotique des valeurs propres d'opérateurs elliptiques dégénérés, Soc. Math. de France, Asterisque, 34-35 (1976), 215-249.

[18] G. Métivier: Valeurs propres des problèms aux limites elliptiques irréguliers, Bull. Soc. Math. France Mem., 51-52 (1977), 125-219.

[19] G. Métivier: Estimation du reste en theorie spectrale, in Seminar Analysis 1982/ 1983, Inst. Math., Berlin (1983), 70-96.

[20] Y. Miyazaki: A sharp asymptotic remainder estimate for the eigenvalues of operators associated with strongly elliptic sesquilinear forms, Japan. J. Math., 15 (1989), 65-97.

[21] Y. Miyazaki: Remarks on irregular open sets and its application to the eigenvalue distribution, preprint.

[22] M.A. Naimark: Linear Differential Operators, Frederick Unger, New York, 1968.

[23] Pham The Lai: Comportement asymptotique du noyau de la résolvante et des valeurs propres d'un opérateur elliptique non nécessairement auto-adjoint, Israel J. of Math. 23 (1976), 221-250.

[24] Pham The Lai: Meilleures estimations asymptotiques des restes de la fonction spectrale et des valeurs propres relatifs au laplacien, Math. Scand. 48 (1981), 538.

[25] M. Reed and B. Simon: Analysis of Operators IV, Academic Press, San Diego, 1978.

[26] R.T. Seeley: A sharp asymptotic remainder estimate for the eigenvalues of the Laplacian in a domain of $\boldsymbol{R}^{3}$, Adv. in Math. 29 (1978), 244-269.

[27] R.T. Seeley: An estimate near the boundary for the spectral function of the Laplace operator, Amer. J. Math. 102 (1980), 869-902.

[28] N. Shimakura: Ordinary Differential Equations, Shōkabo, Tokyo, in Japanese, 1988.

[29] E.M. Stein: Singular Integrals and Differentiability Properties of Functions, Princeton Univ. Press, New Jersey, 1970.

[30] H. Tanabe: On remainder estimates in the asymptotic formula of the distribution of eigenvalues of elliptic operators, Proc. Japan Acad., 48 (1972), 377-380.

[31] J. Tsujimoto: On the asymptotic behavior of spectral functions of elliptic operators, Japan. J. Math., 8 (1982), 177-210. 
[32] J. Tsujimoto: On the remainder estimates of asymptotic formula for eigenvalues of operators associated with strongly elliptic sesquilinear forms, J. Math. Soc. Japan, 33 (1981), 557-569.

[33] J. Tsujimoto: Asymptotic estimates for spectral functions of elliptic operators and its application, RIMS, Kôkyûroku 530, 17-23, in Japanese, 1984.

[34] D.G. Vasil'ev: Two-term asymptotics of the spectrum of a boundary-value problem under an interior reflection of general form, Funktsional. Anal. i Prilozhen., 18, No. 4 (1984), 1-13.

[35] J. Wloka: Partial Differential Equations, Cambridge Univ. Press, Cambridge, 1987.

School of Dentistry, Nihon University

1-8-13 Kanda-Surugadai

Chiyoda-ku

Tokyo

101 Japan 
